Hung, Yost, Xie et al., (CHANG), p. 1

\title{
EcDNA hubs drive cooperative intermolecular oncogene expression
}

2

3 King L. Hung ${ }^{1, \#}$, Kathryn E. Yost ${ }^{1, \#}$, Liangqi Xie ${ }^{2,3,4, \#}$, Sihan Wu ${ }^{5}$, Joshua T. Lange ${ }^{5,6}$, Connor V.

4 Duffy ${ }^{1}$, Katerina Kraft ${ }^{1}$, Jun Tang ${ }^{5}$, Quanming Shi ${ }^{1}$, John C. Rose ${ }^{1}$, M. Ryan Corces ${ }^{1}$, Jeffrey M.

5 Granja $^{1}$, Rui Li ${ }^{1}$, Utkrisht Rajkumar ${ }^{7}$, Robert Tjian $^{3,4}$, Vineet Bafna ${ }^{7}$, Paul S. Mischel ${ }^{5,8}$, Zhe Liu ${ }^{2}$,

6 Howard Y. Chang ${ }^{1,9, *}$

7

${ }^{1}$ Center for Personal Dynamic Regulomes, Stanford University School of Medicine, Stanford, CA 94305, USA.

2 Janelia Research Campus, Howard Hughes Medical Institute, Ashburn, VA, USA

${ }^{3}$ Department of Molecular and Cell Biology, Li Ka Shing Center for Biomedical and Health

2 Sciences, CIRM Center of Excellence, University of California, Berkeley, CA, USA

3 ' Howard Hughes Medical Institute, Berkeley, CA, USA

$4{ }^{5}$ Department of Cellular and Molecular Medicine, University of California at San Diego, La Jolla,

$16{ }^{6}$ Biomedical Sciences Graduate Program, University of California at San Diego, La Jolla, CA,

17 92093, USA

$18{ }^{7}$ Department of Computer Science and Engineering, University of California at San Diego, La

19 Jolla, CA, USA.

$20{ }^{8}$ Department of Pathology, University of California at San Diego, La Jolla, CA, USA.

$21{ }^{9}$ Howard Hughes Medical Institute, Stanford University School of Medicine, Stanford, CA

2294305, USA.

23

24 "These authors share co-first authorship

$25{ }^{*}$ Correspondence should be addressed to: howchang@stanford.edu 
Hung, Yost, Xie et al., (CHANG), p. 2

\section{ABSTRACT}

Extrachromosomal DNAs (ecDNAs) are prevalent in human cancers and mediate high oncogene expression through elevated copy number and altered gene regulation ${ }^{1}$. Gene expression typically involves distal enhancer DNA elements that contact and activate genes on the same chromosome ${ }^{2,3}$. Here we show that ecDNA hubs, comprised of $\sim 10-100$ ecDNAs clustered in the nucleus of interphase cells, drive intermolecular enhancer input for amplified oncogene expression. Single-molecule sequencing, single-cell multiome, and 3D enhancer connectome reveal subspecies of MYC-PVT1 ecDNAs lacking enhancers that access intermolecular and ectopic enhancer-promoter interactions in ecDNA hubs. ecDNA hubs persist without transcription and are tethered by BET protein BRD4. BET inhibitor JQ1 disperses ecDNA hubs, preferentially inhibits ecDNA oncogene transcription, and kills ecDNA+ cancer cells. Two amplified oncogenes MYC and FGFR2 intermix in ecDNA hubs, engage in intermolecular enhancer-promoter interactions, and transcription is uniformly sensitive to JQ1. Thus, ecDNA hubs are nuclear bodies of many ecDNAs tethered by proteins and platforms for cooperative transcription, leveraging the power of oncogene diversification and combinatorial DNA interactions. We suggest ecDNA hubs, rather than individual ecDNAs, as units of oncogene function, cooperative evolution, and new targets for cancer therapy.

\section{INTRODUCTION}

Circular ecDNA encoding oncogenes is a prevalent feature of cancer genomes and potent driver of human cancer progression ${ }^{4-7}$. EcDNAs are covalently closed, double-stranded, and range from $\sim 100$ kilobases to several megabases in size ${ }^{1,8-11}$. EcDNAs lack centromeres and are randomly distributed among daughter cells after each cell division allowing for rapid accumulation and selection of ecDNA variants that drug resistance or other fitness advantage ${ }^{5,12-14}$. ecDNAs can evolve over time from submicroscopic episomes to large double minutes, and that these extrachromosomal elements can re-integrate into chromosomes and form tandem repeats termed homogeneously staining regions (HSRs) ${ }^{15-19}$. ecDNA possess increased chromatin accessibility and lack higher order chromatin compaction ${ }^{1,20}$, and encompass the endogenous oncogene enhancer elements ${ }^{21}$. ecDNA exists outside the normal chromosomal context by definition, and its spatial organization in the nucleus is poorly understood. Notably, ecDNAs can cluster during cell division or after DNA damage with unclear biological consequences ${ }^{22-24}$.

The MYC oncogene on human chromosome $8 q 24$ is a hotspot for somatic DNA rearrangements in cancer ${ }^{25}$, and nearly $30 \%$ of $M Y C$ amplifications in human cancers exist as ecDNA ${ }^{5}$, typically encompassing $M Y C$ and 5' portion of PVT1 (plasmacytoma variant transcript 
1). PVT1, located $55 \mathrm{~kb} 3$ ' of $M Y C$, is a recurrent breakpoint in human cancers ${ }^{26,27}$. Structural rearrangements in PVT1 often lead to the transcriptional activation of $M Y C$, and historically led the way for the recognition of $M Y C$ as an oncogene ${ }^{27,28}$. The PVT1 gene encodes a long

72 noncoding RNA and contains multiple intragenic enhancers that normally interact with the PVT1

73 promoter. However, when PVT1 promoter is mutated or silenced, PVT1 intragenic enhancers can

74 activate $M Y C$ instead ${ }^{26}$. The dynamic competition between $M Y C$ and $P V T 1$ promoters implicated

75 PVT1 promoter as a tumor suppressor DNA boundary element. Here we examine the spatial,

76 epigenetic, and transcriptional dynamics of oncogenic ecDNAs, focusing on MYC-PVT1 in human

77 cancer cells, and reveal ecDNA hubs as combinatorial enhancer platforms for cooperative 78 oncogene transcription.

\section{RESULTS}

\section{ecDNA hubs are predominant sites of oncogene transcription}

To understand the 3D context of ecDNA during transcription, we visualized the localization of ecDNAs in the nucleus during interphase using DNA fluorescence in situ hybridization (FISH) ${ }^{29}$ (Figure 1a). We designed probes targeting corresponding oncogenes and surrounding sequences amplified on ecDNAs in multiple ecDNA-containing cell lines including PC3 (MYCamplified; prostate cancer), COLO320-DM (MYC-amplified; colorectal carcinoma), HK359 (EGFR-amplified; glioblastoma multiforme), and SNU16 (MYC- and FGFR2-amplified; gastric cancer) $)^{1}$ (Supplemental Figure 1a). To validate that these sequences are located on ecDNA molecules, we performed DNA FISH on metaphase spreads to confirm the presence of tens to hundreds individual extrachromosomal DNAs per cell based on DNA FISH signal located outside of metaphase chromosomes (Supplemental Figure 1b). In a subset of cell lines, we also employed two-color DNA FISH to interrogate a neighboring control locus in cis that is not amplified on ecDNA (Supplemental Figure 1a); chromosomal copies of the oncogene appear as paired dots with neighboring chromosomal loci proximal to each other (Figure 1a) while ecDNAs have a single color. Two-color FISH experiment in an ecDNA-negative cell line, HCC1569, consistently showed paired two-color signals of similar sizes as expected from the chromosomal loci

98 (Supplemental Figure 1c). In contrast, in all ecDNA-positive cancer cells we assessed, DNA

99 FISH signal for ecDNAs was largely restricted to specific areas of the nucleus of interphase cells

100 despite arising from tens to hundreds of individual ecDNA molecules, suggesting that ecDNAs

101 strongly clustered with one another, a feature we term ecDNA hubs (Figure 1a). These ecDNA

102 hubs occupy a much larger space than neighboring chromosomal segments of the same size, 
Hung, Yost, Xie et al., (CHANG), p. 4

103 suggesting that they are composed of many ecDNA molecules clustering tightly in space. We

104 used the pair autocorrelation function $\mathrm{g}(\mathrm{r})$ (Methods) to measure the spatial distribution of ecDNA

105 hubs. $g(r)$ estimates the probability of detecting another ecDNA signal at increasing distances

106 from the viewpoint of an index ecDNA signal and is equal to 1 for a uniform, random distribution.

107 This quantification showed a significant increase in ecDNA clustering over short distances (0-40

108 pixels, 0-1.95 microns, Figure 1b), with all cell lines and oncogenes displaying increased 109 autocorrelation compared to a simulated random distribution (Methods). EcDNA clusters were 110 much larger than diffraction limited spots ( 0.3 microns), consistent with co-localization of multiple 111 ecDNA copies within individual clusters. To confirm these findings in live cells, we generated a 112 Tet-operator (TetO) array knock-in of the MYC ecDNA in COLO320-DM cells, and labeled ecDNA 113 with TetR-eGFP (TetO-eGFP COLO320-DM) (Figure 1c, Methods). Live cell imaging revealed 114 multiple bright and locally accumulated signal in the nucleus likely corresponding to ecDNA hubs 115 composed of clustered ecDNA molecules, whereas the TetR-eGFP signal is homogeneously 116 distributed in the parental cell line without the TetO array (Figure 1c, Supplemental Figure 1d,

117 Supplemental Movie 1). EcDNA hubs are dynamic in living cells; they both move and change 118 shape with time. We note that TetR-eGFP labeled ecDNA hubs are spatially more compact in 119 living cells than in DNA FISH studies of fixed cells, likely due to the fact that the TetO array is not 120 integrated in all ecDNA molecules, as well as potential differences induced by denaturation during 121 DNA FISH. These results suggest that ecDNA clustering occurs across various cancer types with 122 different oncogene amplifications.

123 To assess the transcriptional activity of ecDNA hubs, we combined DNA and nascent RNA 124 FISH in the PC3 and COLO320-DM cell lines to visualize actively transcribing MYC alleles as 125 colocalized DNA and RNA FISH signal, using a short $100 \mathrm{~kb}$ Oligopaint DNA FISH probe to enable 126 quantification of individual ecDNA molecules within ecDNA hubs (Figure 1d, Supplemental 127 Figure 1a,e-f, Methods). We quantified colocalized RNA and DNA FISH signals to compute the 128 probability of MYC transcription from each ecDNA molecule in a snapshot of time (Methods); this 129 measure accounts for the copy number of ecDNA template and subsumes the frequency and 130 duration of MYC transcription. In the PC3 cell line, we were able to more confidently distinguish 131 chromosomal MYC transcription from ecDNA-derived transcription due to lower ecDNA copy 132 numbers (Figure 1d). Interestingly, the majority of nascent MYC mRNA transcripts colocalized 133 with ecDNA clusters rather than the chromosomal locus and transcription probability is 134 significantly higher from ecDNA relative to the chromosomal locus (Figure 1d,e). Quantification 135 of nascent MYC transcription from ecDNAs vs ecDNA clustering (using the autocorrelation 136 function of ecDNA FISH signal, Methods) showed a significant correlation between MYC 
137 transcription probability and ecDNA clustering $(R=0.48-0.55, p<0.05$ for both PC3 and COLO320-

$138 \mathrm{DM}$, Figure 1f left). EcDNA clustering is a better predictor of ecDNA transcription probability than 139 ecDNA copy number ( $\mathrm{n}=14-50$ in PC3, $\mathrm{n}=49-441$ in COLO320-DM, $R=0.16-0.42$, Figure $1 \mathrm{f}$ right).

140 Thus, each ecDNA molecule is more likely to transcribe the oncogene when more ecDNA copies

141 are in the same cell, especially in the form of ecDNA hubs.

\section{Single-cell co-variation identifies ecDNA enhancers associated with potent oncogene} expression

To understand regulation of oncogene expression on ecDNAs, we set out to identify regulatory elements on ecDNAs that correlate with high oncogene expression. While previous data suggest that ecDNAs contribute to cancer cell heterogeneity ${ }^{6,9}$, the chromatin regulatory

148 landscape of ecDNAs and its relationship to oncogene transcription has not been studied on a 149 single-cell level. To address this, we focused on a pair of colorectal cancer cell lines, COLO320-

150 DM and COLO320-HSR, which were derived from the same patient tumor and therefore contain 151 highly similar genetic backgrounds except for the context in which MYC is amplified ${ }^{17}$. MYC is 152 amplified on ecDNA in COLO320-DM cells versus tandem chromosomal amplicons (HSRs) in 153 COLO320-HSR cells (Supplemental Figure 2a). Given the heterogeneous nature of ecDNAs 154 across COLO320-DM cells, we hypothesized that we could exploit the cell-to-cell variation in ecDNA sequence to identify regulatory elements whose activity positively predicts potent ecDNA oncogene expression in individual cells. We took a single-cell multiomic approach and performed

157 a droplet-based, paired assay for transposase-accessible chromatin using sequencing (ATAC$158 \mathrm{seq}$ ) and RNA sequencing in the same single cell (Supplemental Figure 2b,c, Methods) and 159 obtained paired transcriptomic and chromatin accessibility profiles from a total of 72,049 cells. 160 Cells were first visualized with uniform manifold approximation and projection (UMAP) ${ }^{30}$ 161 independently based on either transcriptomic or chromatin accessibility profiles (Methods). 162 UMAPs of either single-cell ATAC-seq or single-cell RNA-seq data showed separate clustering of 163 COLO320-DM and COLO320-HSR cell lines as expected (Figure 2a). We then integrated the 164 transcriptomic and chromatin accessibility profile for each cell to interrogate how chromatin 165 accessibility covaries with gene expression. For each cell, we calculated a gene accessibility 166 score for MYC, which incorporates ATAC-seq signals from the gene body and those from distal 167 regulatory elements ${ }^{31}$ (Methods). Accessibility scores for MYC increased with RNA expression 168 (Supplemental Figure 2d; Pearson $\mathrm{R}=0.25, \mathrm{p}<2.2 \mathrm{e}-16$ ). RNA expression as well as 169 accessibility scores for MYC were highly heterogeneous in the ecDNA MYC-amplified COLO320170 DM cell population relative to the chromosomal HSR MYC-amplified COLO320-HSR population 
Hung, Yost, Xie et al., (CHANG), p. 6

171 (Figure 2b). These observations suggest that variable activities of regulatory elements may

172 explain cell-to-cell variation in oncogene expression by ecDNAs.

173 To identify active regulatory elements on ecDNAs in cells that express high levels of MYC,

174 we assigned cells into 20 bins based on MYC RNA levels and adjusted ATAC-seq signals from

175 each bin using copy numbers calculated from background coverage to perform pairwise testing

176 of differential ATAC-seq peaks between the top and the bottom RNA expression bin

177 (Supplemental Figure 2b, Methods). A comparison of ATAC-seq coverage tracks suggested 178 that high MYC-expressing COLO320-DM cells contain both higher ecDNA copy number based 179 on increased background signal as well as a number of increasingly accessible elements, while 180 chromosomal HSRs displayed similar chromatin accessibility profiles in the top and bottom RNA 181 bins of COLO320-HSR cells (Figure 2c). Differential peak analysis identified 47 active elements 182 on ecDNA that were strongly associated with high MYC expression compared to 5 active 183 elements on chromosomal HSRs (Figure 2d). These findings suggest that increased MYC 184 transcription is associated with increased chromatin accessibility at numerous distal regulatory 185 elements in the context of ecDNA. The most significantly upregulated DNA elements (named by 186 their distance to the MYC promoter) demonstrated accessibility that scaled with MYC mRNA 187 expression when accounting for DNA copy number only in the ecDNA context (Supplemental 188 Figure 2e, zoomed in). Notably, significantly active elements were distributed throughout the 189 entire amplified region. Most of these active elements were concentrated in the interval between 190 FAM84B and CASC8, consistent with increased accessibility of regulatory elements 5' of the MYC 191 coding sequence across colon adenocarcinomas ${ }^{32}$. As expected, cells with high MYC expression 192 tend to have higher ecDNA copy numbers; however, we note that there is a high level of variability 193 in ecDNA copy number for cells with similar levels of MYC RNA output, suggesting that copy 194 number alone does not fully predict RNA expression (Supplemental Figure 2f,g). Importantly, 195 the decreased accessibility at regulatory elements in the low RNA bin of COLO320-DM is not 196 likely to be caused by differences in data quality, as we did not observe a decrease in overall TSS 197 enrichment (Supplemental Figure 2h). Moreover, accessibility profiles of high and low MYC RNA 198 bins in COLO320-HSR cells are highly similar, demonstrating that the strong co-variation of 199 regulatory elements with MYC expression and their heterogeneous activities within COLO320200 DM cells may be a unique feature of ecDNA (Figure 2c,d). Together, these results indicate that 201 differential enhancer usage may be key to understanding cellular heterogeneity in ecDNA 202 oncogene expression. 
Hung, Yost, Xie et al., (CHANG), p. 7

204 Combinatorial intermolecular contacts between enhancers and promoters drive ecDNA 205 diversification and oncogene expression.

With the discoveries that (i) ecDNA hubs in the nucleus are associated with active oncogene expression and (ii) oncogene expression by ecDNAs is linked to differential regulatory elements, we next interrogated whether these differential elements participate in novel enhancerpromoter interactions among potentially distinct ecDNA molecules. While one may predict that each ecDNA molecule must contain all of the sequence elements (enhancers, promoters, etc.) to promote oncogene expression in cis, the recognition of ecDNA hubs where multiple ecDNA molecules come into spatial proximity raises the possibility that DNA regulatory elements may cooperate among several ecDNAs to enable oncogene expression. In this second scenario, it may be possible to observe selection for ecDNA structures which lack canonical cis regulatory elements necessary for oncogene expression as well as ecDNA structures without coding oncogene elements if these distinct molecules can cooperate within transcriptionally active hubs. Here we integrate whole genome sequence analysis, single-molecule DNA sequencing, and 3D enhancer connectome mapping to distinguish between these two scenarios.

To reconstruct ecDNA molecules in COLO320-DM cells, we obtained whole-genome sequencing data and applied AmpliconArchitect, a computational tool which uses both copy number variation and structural variant analysis of short sequencing reads to reconstruct DNA amplicons arising from ecDNAs and other complex rearrangements ${ }^{5,6,33}$. We identified multiple

223 "subspecies" of ecDNA amplicons resulting from the rearrangement of the MYC locus in 224 COLO320-DM cells with a range of copy numbers (Supplemental Figure 3a,b). Notably, we 225 detected abundant ecDNAs which lacked either the MYC coding sequence and contained only 226 regulatory DNA elements, or conversely, contained a truncated MYC coding sequence and lacked 227 distal regulatory elements (Supplemental Figure 3b). Next, we used nanopore-based single228 molecule sequencing to obtain long contiguous ecDNA reads (mean $9 \mathrm{~kb}$, maximum $201 \mathrm{~kb}$; 229 Supplemental Figure 3c). We observed high concordance between structural variants detected 230 by long and short-read sequencing (Supplemental Figure 3d) and confirmed that one of the 231 most abundant rearrangements of the MYC locus generates an $86 \mathrm{~kb}$ amplicon that comprises 232 the promoter and exon 1 of noncoding RNA PVT1 fused to exons 2 and 3 of MYC (Figure 2e). 233 Here the PVT1 promoter replaces the MYC promoter to generate a PVT1-MYC fusion transcript 234 and a new 5' UTR for MYC, which is hypothesized to overcome PVT1 and MYC promoter 235 competition $^{26}$. Previous studies have demonstrated that a functional protein isoform of MYC can 236 initiate from a start codon in exon 2, suggesting that transcripts derived from the fusion PVT1237 MYC transcript generate functional MYC protein ${ }^{34,35}$. However, the amplicon containing the PVT1- 
Hung, Yost, Xie et al., (CHANG), p. 8

MYC fusion in COLO320-DM cells is not predicted to be covalently linked to many of the active regulatory elements located 5' of the MYC coding sequence. We also confirmed multiple junctions present in a predicted $\sim 1.58 \mathrm{Mb}$ amplicon with full-length $M Y C$ coding sequence that retains many distal regulatory elements but is predicted to be present at a lower copy number (64 instead of 102 for the $86 \mathrm{~kb}$ amplicon). We observed multiple single-molecule sequencing reads spanning an $8.5 \mathrm{~kb}$ segment predicted to join two opposite ends of the larger amplicon segment (Figure 2e).

Our results suggest a high degree of heterogeneity among ecDNA molecules in COLO320-DM, with two major subspecies possessing unique variants of the MYC coding sequence as well as distinct connectivity to putative regulatory elements. Previous studies have also noted the existence of the PVT1-MYC fusion in COLO320-DM cells, originally as a 5' structural abnormality of the MYC gene observed by Northern blot analysis ${ }^{36-38}$ and more recently with sequencing based methods ${ }^{39}$. We confirmed by RNA-seq analysis that the majority (79\%) of the MYC mRNA transcripts in COLO320-DM cells arise from the PVT1-MYC fusion present on the $86 \mathrm{~kb}$ amplicon (predicted to be $61 \%$ of $M Y C$ ecDNA by DNA copy number) (Figure $\mathbf{2 f}$ ). Single-cell transcriptomic co-variation analysis also validated PVT1-MYC transcription in COLO320-DM; reads mapping to PVT1 positively correlated with reads mapping to MYC in COLO320-DM cells but not COLO320-HSR cells (Pearson R = 0.996 for COLO320-DM, R = 0.909 for COLO320-HSR; Supplemental Figure 3e). Thus, in a cancer cell line harboring a variety of ecDNA species, oncogene output appears to be preferentially driven by a specific ecDNA subspecies. Moreover, the rearrangement that creates the $86 \mathrm{~kb}$ amplicon excludes known MYC enhancers both 5' of the MYC transcriptional start site or 3' in the PVT1 locus, raising the question of whether and how ecDNA-encoded PVT1-MYC accesses distal enhancers.

Next, we mapped active enhancers and their target genes in COLO320-DM cells using HiChIP, a protein-directed 3D genome conformation assay ${ }^{40,41}$, targeting histone H3 lysine 27 acetylation (H3K27ac) associated with active enhancers and enriched on ecDNA as observed by immunofluorescence in metaphase cells ${ }^{1}$. These results first provided independent confirmation of the $86 \mathrm{~kb}$ PVT1-MYC amplicon, which is evident as a tightly interacting core on the HiChIP map with increased vertex signal following correction for copy number variation with square root coverage normalization (Figure 2g, arrow, Methods). Second, we identified a number of active enhancer elements identified by single-cell ATAC-seq originally located 5 ' of the MYC gene with significant contact to the PVT1/PVT1-MYC promoter (Figure 2g, Supplemental Figure 3f). We noted that while the canonical MYC promoter participates in several focal enhancer contacts (Supplemental Figure $\mathbf{3 g}$ ), HiChIP signal at the PVT1 promoter is elevated across the entirety 
Hung, Yost, Xie et al., (CHANG), p. 9

272 of the amplified region, supported by a uniform density of highly significant chromatin contacts

273 (Figure 2g). While several loops overlap structural rearrangements identified by long-read 274 sequencing, many high-confidence loops identified by HiChIP are independent of structural 275 arrangements, suggesting they represent true enhancer-promoter contacts (Supplemental

276 Figure 3d). Collectively, these results suggest an extensive degree of ecDNA primary sequence 277 diversification, including structural variants that would be predicted to limit contact in cis with distal 278 regulatory elements. However, robust transcription associated with combinatorial and 279 intermolecular enhancers usage in within ecDNA hubs led us to speculate that multiple species 280 of ecDNA molecules may cooperate when in spatial proximity to achieve robust transcription of 281 cargo genes.

BRD4 bridges ecDNA hubs and drives hub oncogene transcription

The extensive long-range and H3K27ac-associated DNA contacts raised the possibility that BET proteins may be involved in transcription from ecDNA hubs. Bromodomain and extraterminal domain (BET) proteins are chromatin reader proteins that recognize H3K27ac and are intricately involved in enhancer function. Genomic regions with multiple contiguous enhancers, termed super enhancers or stretch enhancers, are recognized based on their extensive decoration by H3K27ac, transcription coactivator complex Mediator, and BET proteins such as BRD4 ${ }^{42,43}$. The MYC gene is flanked by tissue-specific super enhancers in certain cancers and BRD4 occupancy as well as MYC transcription are highly sensitive to the BET inhibitor JQ1 ${ }^{44}$, which displaces BET proteins from $\mathrm{H} 3 \mathrm{~K} 27 \mathrm{ac}^{45}$. We also previously showed that BRD4 occupancy marked the winner in promoter competition between $P V T 1$ and $M Y C^{26}$. To determine the role of

294 BET proteins in ecDNA-derived transcription, we examined BRD4 occupancy at the MYC locus 295 in both COLO320-DM and COLO320-HSR cells. Endogenous epitope tagging of BRD4 combined 296 with ecDNA labeling with TetR-eGFP showed that BRD4 colocalized with ecDNAs in COLO320297 DM cells (Figure 3a). This colocalization of GFP and BRD4 signals was not observed in cells 298 without TetO integration (Supplemental Figure 4a). Chromatin immunoprecipitation and 299 sequencing (ChIP-seq) of H3K27ac, BRD4, and ATAC-seq in COLO320-DM and COLO320-HSR 300 cells showed that indeed H3K27ac peaks, marking active ecDNA enhancers, are also occupied 301 by BRD4, including many active elements associated with high MYC transcription and in contact 302 with the PVT1-MYC promoter (-1132E, -1087E, -679E, -655E, -401E, -328E, -85E) 303 (Supplemental Figure 4b). Importantly, the PVT1 promoter is one of the highest sites of BRD4 304 occupancy in ecDNA containing cells (COLO320-DM) but not in cells with chromosomal 305 amplification (COLO320-HSR) (Figure 3b, Supplemental Figure 4c). Whole genome 
sequencing showed that HSR amplicons in COLO320-HSR do not contain the PVT1 promoter, as evidenced by reduced whole genome sequencing coverage (Figure $3 \mathbf{b}$, WGS track). Thus, ecDNAs and chromosomal amplicons in this cell line pair have adopted two diametrically opposite strategies to inactivate the tumor suppressor PVT1 promoter - cooption of PVT1 promoter to drive MYC transcription in ecDNA versus deletion of PVT1 promoter in chromosomal MYC amplicons.

Next, we evaluated the consequences of BET inhibitor JQ1 on ecDNA hubs, the dominant site of MYC transcription in COLO320-DM. Treatment with 500 nM JQ1 dramatically dispersed ecDNA hubs in COLO320-DM after 6 hours (Figure 3c); large ecDNA hubs split into multiple small ecDNA signals within the nucleoplasm, as reflected in reduced autocorrelation $\mathrm{g}(\mathrm{r})$ across multiple length scales (Figure 3c). However, ecDNA dispersal by JQ1 appears to be a highly specific effect. Transcription inhibition by either the RNA polymerase II inhibitor alpha-amanitin or treatment with 1,6-hexanediol, a hydrophobic compound that can dissolve certain nuclear condensates $^{46}$, both of which robustly reduce MYC transcription (Supplemental Figure 5a,b), did not affect ecDNA hubs (Figure 3c, Supplemental Figure 5c). Live cell imaging with TetOGFP COLO320-DM cells revealed that JQ1 causes ecDNA hubs to disperse after $\sim 30$ minutes of drug treatment while ecDNA hubs in control cells remain intact (Figure 3d, Supplemental Movie 2,3). Together, these results suggest that bromodomain-H3K27ac interaction of BET proteins is essential for ecDNA clustering.

We next examined the effect of JQ1 on ecDNA-derived oncogene transcription. JQ1 treatment strongly reduced MYC transcription probability by four-fold per ecDNA copy, as evidenced by joint nascent RNA and DNA FISH (Figure 3e, Supplemental Figure 5a). Because BET proteins are also involved in MYC transcription from chromosomal DNA, we further compared the effect of JQ1 on COLO320-DM vs COLO320-HSR cells. BRD4 ChIP-seq showed that JQ1 treatment equivalently dislodged BRD4 genome-wide in these isogenic cells

330 (Supplemental Figure 5d). Nonetheless, treatment with $500 \mathrm{nM}$ JQ1 preferentially lowered MYC 331 mRNA level in COLO320-DM cells, a dose which had no significant effect on MYC mRNA level 332 in COLO320-HSR cells (Figure 3f). Dose titration of JQ1 confirmed the preferential killing of 333 COLO320-DM cells over HSR cells (Figure $\mathbf{3 g}$ ). These results demonstrate a unique dependence 334 on BET proteins for ecDNA hub formation; disruption of ecDNA hubs cause preferential suppression of MYC oncogene transcription and death of ecDNA-bearing cancer cells.

In contrast to the ability of BETi to inhibit ecDNA transcription, we found that ecDNAs

337 appear resistant to targeting of individual enhancers. We and others have previously used 338 CRISPR interference (CRISPRi) with targeted catalytically dead Cas9 fused to KRAB silencer 339 domain to identify functional MYC enhancers in the chromosomal context ${ }^{26,41,47}$. CRISPRi of the 
Hung, Yost, Xie et al., (CHANG), p.11

PVT1 promoter, but not MYC promoter, indeed reduced PVT1-MYC transcription and total MYC mRNA level in COLO320-DM, confirming the promoter cooption from the 86kb ecDNA subspecies and serving as positive control (Supplemental Figure 5e). In contrast, individually targeting 6 enhancers with high BRD4 occupancy on ecDNA did not significantly reduce bulk MYC mRNA levels (Supplemental Figure 5e,f). These results suggest that ecDNA dispersal may be a more effective strategy to overcome highly cooperative oncogene enhancers in ecDNA hubs.

\section{EcDNA hubs support intermolecular cross regulation of two oncogene loci}

As several lines of evidence presented above suggest that ecDNA hubs may promote intermolecular enhancer-promoter interactions, we investigated whether these interactions can be precisely mapped and perturbed. Due to the overlap of DNA segments that compose ecDNA amplicons in the COLO320-DM cell line, uniquely mapping enhancer elements to distinct molecules is challenging. To overcome this, we focused on a human gastric cancer cell line, SNU16, which contains two major types of ecDNAs. One type of ecDNA contains a MYC amplicon derived from chromosome 8 and the other contains an FGFR2 amplicon derived from chromosome 10. Image analysis of dual-color metaphase FISH in SNU16 cells with EcSeg showed that these two amplicons are located on distinct molecules in the majority of ecDNAs ( $\sim 35 \%$ of all ecDNA molecules contain MYC only, and $\sim 60 \%$ contain FGFR2 only; Figure 4a,b $)^{48}$, which was also supported by analysis of whole-genome sequencing by AmpliconArchitect (Supplemental Figure 6a,b). In contrast, MYC and FGFR2 ecDNAs intermingle with each other during interphase in hubs as demonstrated by increased colocalization in dual-color interphase FISH (Figure 4c,d). These data led us to hypothesize that ecDNA hubs in SNU16 may allow intermolecular interactions between MYC-bearing and FGFR2-bearing ecDNAs. To test this idea, we performed H3K27ac HiChIP on SNU16 cells to map enhancer-promoter interactions. Because a small percentage of ecDNA molecules appeared to contain both MYC and FGFR2 ( 5\%, Figure 4b), we used AmpliconArchitect to identify regions that were predicted to be fused on ecDNA, where we observe a high density of HiChIP signal potentially resulting from cis interactions (Figure 4e, dashed box highlighting translocation). Outside of this region, we identified intermolecular contacts between active enhancers from the MYC super enhancer to FGFR2 promoter, as well as enhancer contacts from FGFR2 amplicon back to MYC (Figure 4e, arrows). These intermolecular interactions remain prominent following correction for copy number variation with square root coverage normalization.

To assess the effects of perturbing intermolecular enhancer-promoter interactions on each respective oncogene, we stably expressed dCas9-KRAB in SNU16 cells (SNU16-dCas9-KRAB) 
374 and delivered lentiviral pools of sgRNA guides targeting regulatory elements located on either

375 MYC-bearing ecDNAs or FGFR2-bearing ecDNAs (Supplemental Figure 6c). After guide 376 infection and antibiotic selection, cells were labeled with MYC or FGFR2 mRNA targeting FISH

377 probes, signal was amplified with PrimeFlow, and RNA expression of each oncogene was 378 analyzed by flow cytometry. We sorted on cell fractions that had negative, low or high expression 379 of either oncogene, extracted genomic DNA and performed targeted sequencing of the lentiviral 380 guide pools (Supplemental Figure 6c). Based on guide abundances in each cell fraction 381 compared to unsorted cells, we calculated a combined repression score that summarizes the 382 degree to which each guide is enriched in cells with low oncogene expression and depleted in 383 cells with high oncogene expression (Methods, Supplemental Figure 6c). A high repression 384 score would suggest that the targeted enhancer normally upregulates oncogene expression. 385 Using this approach, we found that CRISPRi of a subset of H3K27ac- and BRD4-bound enhancer 386 regions 5' of MYC as well as MYC TSS inhibited MYC RNA expression, while CRISPRi of loci not 387 bound by H3K27ac or BRD4 had no effect on expression (Figure 4e, bottom panel, 388 Supplemental Figure 6d). CRISPRi of enhancers on MYC ecDNAs had minimal effect on 389 FGFR2 expression. Conversely, CRISPRi of 5' and intragenic enhancers of FGFR2 led to 390 decreases in both FGFR2 in cis and MYC expression in trans (Figure 4e, right panel, 391 Supplemental Figure 6d), suggesting that enhancers derived from the FGFR2 locus may 392 activate transcription from MYC ecDNAs. Given that a small percentage of ecDNAs contain 393 sequences from both the MYC and the FGFR2 loci (Figure 4b,e), two of the intergenic FGFR2 394 enhancers that affect MYC transcription lie in the predicted translocated region, and thus could 395 be either cis- or trans-acting. However, the FGFR2 intragenic enhancer is required for MYC 396 expression and is located outside of the predicted fusion circles and is a bona-fide trans-acting 397 enhancer (Figure 4e). This FGFR2 ecDNA enhancer is also located within a broad region with 398 increased intermolecular interactions with MYC ecDNAs as shown by increased HiChIP signals. 399 Finally, treatment with the BET protein inhibitor JQ1 led to concomitant reduction in both MYC 400 and FGFR2 expression (Figure 4f). These observations suggest that colocalization of MYC-and 401 FGFR2-encoding ecDNAs in hubs may be important for the expression of both oncogenes. DISCUSSION In this study, we show that oncogene-carrying ecDNAs in cancer cells strongly colocalize 405 in clusters that associate with high levels of transcription, a phenomenon we term ecDNA hubs. 406 This local congregation of ecDNAs promotes novel enhancer-promoter interactions and 407 oncogene expression (Figure $\mathbf{4 g}$ ). In turn, variable usage of these enhancers across molecules 
Hung, Yost, Xie et al., (CHANG), p.13

408

409

410

411

412

413

414

415

416

417

418

419

420

421

422

423

424

425

426

427

428

429

430

431

432

433

434

435

436

437

438

439

440

441

strongly contributes to cell-to-cell heterogeneity in oncogene-driven programs. Unlike chromosomal transcription hubs which are restricted to local cis regulatory elements, chromatin conformation data and CRISPR interference suggest that ecDNA hubs may also involve trans regulatory elements arising on distinct ecDNA molecules. This discovery has profound implications in 1) how ecDNAs undergo selection and 2) how rewiring of oncogene regulation on ecDNA contributes to oncogenic transcription and cancer cell heterogeneity.

\section{EcDNA hubs in oncogene selection and cancer cell heterogeneity}

EcDNA molecules are products of stringent genetic selection which are able to drive high levels of oncogene expression outside of the normal chromosomal context and provide a fitness advantage to cancer cells that harbor them. Given that ecDNA has been separated from the 3D genomic context of its chromosomal origin, it has been proposed that the co-selection of oncogenes and enhancers shapes ecDNA amplicon structures ${ }^{21}$. In this study, we presented evidence for intermolecular interactions among ecDNA molecules carrying distinct enhancer elements. With this new observation, we propose a two-level model for oncogene-enhancer coselection. The first level of co-selection occurs to individual ecDNAs; molecules that possess functional enhancers can promote oncogene expression and provide better fitness to cancer cells compared to ones that do not. The second level of co-selection occurs to the repertoire of ecDNAs in hubs. In other words, we predict that individual ecDNA molecules are not required to contain all of the enhancers necessary for promoting oncogene expression; rather, they exist as part of an ecDNA hub that facilitates chromatin interactions among a diverse repertoire of regulatory elements and promotes interactions between the target oncogene and functional enhancers which may be located on distinct molecules. This model raises the intriguing concept that winning the clonal competition among cancer cells occurs through clonal cooperation among ecDNA molecules. This type of evolutionary dynamics has been documented in viruses, where cooperation of a mixture of specialized variants outperforms a pure population of wild type virus $^{49,50}$. Furthermore, our ecDNA cooperation model predicts that mutations on individual molecules may be better tolerated if functional elements are present on other molecules in a hub. If true, this tolerance may increase ecDNA sequence diversity and permit rare mutations, including those that confer resistance to therapies. Others have previously reported ecDNA mutational diversity and rapid response to environmental changes ${ }^{51}$, though further investigation is needed to measure mutational diversity in functional enhancers on ecDNAs.

440 Our study shows that enhancer usage can be highly variable on ecDNAs, which associates with cancer cell heterogeneity in oncogene activity. This may be attributed to 
Hung, Yost, Xie et al., (CHANG), p.14

442 differential enhancer-promoter interactions which occur in the context of ecDNA hubs. As dozens

443 of ecDNA molecules can cluster together in many possible spatial configurations, oncogene

444 promoters may have a greater opportunity to "sample" various enhancers via novel enhancer-

445 promoter interactions. When different ecDNAs arise from two different chromosomes, such as in

446 SNU16 cells, they intermingle in ecDNA hubs that enable ectopic enhancer-promoter interactions

447 among oncogene loci that normally do not occur on linear chromosomes. We speculate that these

448 differential interactions contribute to the highly variable enhancer activities and enhancer rewiring

449 on ecDNAs.

$P V T 1$ and $M Y C$ regulation

PVT1, specifically its promoter element, emerges as a strong selective pressure that shapes ecDNA and HSR amplicons. Most copies of the MYC-PVT1 amplicon in COLO320-HSR cells have focal deletions of the PVT1 promoter, suggesting that a PVT1 promoter deletion was either an early mutation or provided a strong selective advantage. This observation is consistent with experimental data that a focal PVT1 promoter deletion, as small as six bases, can activate MYC expression in the chromosomal context and confer a growth advantage ${ }^{26}$. A different strategy is adopted in COLO320-DM cells, in which a rearrangement fuses the PVT1 promoter to the MYC oncogene; the fusion PVT1-MYC transcript becomes the dominant MYC mRNA in COLO320-DM cells. These appear as two divergent strategies for overcoming promoter competition between PVT1 and MYC and amplifying MYC expression.

\section{Potential mechanisms of ecDNA hub formation}

What may be the forces that drive ecDNA hub formation? Transcriptional hubs are thought to be nuclear condensates formed by high concentration of transcription factors, mediator, and RNA polymerase II through interaction of low complexity protein sequences and RNA ${ }^{52-54}$. Small, transient hubs of several hundred protein molecules are distinguished from stable condensates formed by liquid-liquid phase separation that are 10-20 times larger and restrict the movement of molecules into and out of the condensate (reviewed by ${ }^{55}$ ). Small transient transcriptional hubs 470 are necessary for gene transcription ${ }^{56}$, and we speculate that ecDNA hubs are a kind of 471 transcriptional hub mediated by protein-protein interaction involving BRD4 and likely additional 472 proteins. MYC ecDNA hubs are not disrupted by transcriptional inhibition with alpha-amanitin nor 473 by 1,6-hexanediol, suggesting that ecDNA hubs do not depend on RNA polymerase II or specific 474 IDR-IDR interactions sensitive to hexanediol such as Mediator $1^{46}$. BRD4 bromodomains have 475 also been report to mediate recruitment into transcription hubs independent of IDR-IDR 
Hung, Yost, Xie et al., (CHANG), p.15

476 interactions ${ }^{57,58}$, and BRD4-NUT translocation and overexpression in rare human cancers can

477 cause interchromosomal interactions ${ }^{59}$. Interestingly, McSwiggen et al. have previously proposed

478 a nuclear compartmentalization model for Herpes Simplex Virus replication compartments in

479 which accessible viral DNA outcompetes host chromatin for the transcriptional machinery and

480 creates high local concentrations of RNA polymerase ${ }^{60}$. We speculate that ecDNA hubs may

481 exploit via a similar mechanism, wherein accessible ecDNAs sponge up BRD4 and DNA-binding

482 proteins locally to create this uneven spatial distribution.

483 BET proteins are required for ecDNA hub maintenance and oncogene transcription in the

484 COLO320-DM and SNU16 models. As BET proteins can normally concentrate accessible DNA, 485 exclude heterochromatin, and mediate long-range enhancer-promoter communication ${ }^{58,61}$, our 486 results suggest that ecDNA hubs may coopt endogenous mechanisms of long-range gene looping 487 within chromosomes to promote intermolecular chromatin interactions in ecDNA ensembles. 488 While we have focused on MYC in this study, our model predicts that other ecDNA oncogenes 489 may also exploit their endogenous enhancer mechanisms to operate in ecDNA hubs. As others 490 have shown that functional enhancers are co-selected with EGFR on ecDNAs in glioblastoma ${ }^{21}$, 491 we speculate that proteins that mediate endogenous enhancer-EGFR interactions could be 492 involved in ecDNA hub maintenance as well.

\section{Implications for cancer cell evolution and therapeutic opportunities}

495 Our results suggest that ecDNA hubs provide a palatable explanation for well-known 496 tendency of a subset of ecDNA+ cancer cells to develop HSRs. A recent study has shown that 497 double-strand breaks in ecDNAs can trigger aggregation, micronucleus formation, and 498 reintegration into chromosomal $\mathrm{HSRs}^{24}$. Rather than independently suffering concurrent DNA 499 breaks and integrating into the same chromosomal locus, ecDNAs that are spatially proximal in 500 hubs could enable correlated DNA breaks ${ }^{62}$ and concentrated DNA cargo, creating a potential set 501 up for HSR formation. Finally, ecDNA hubs may impact ecDNA replication and segregation. 502 Previous work has demonstrated that ecDNAs are transmitted into daughter cells in clusters 503 during mitosis ${ }^{23}$. Interestingly, papillomavirus episomes hitchhike into daughter cells during 504 mitosis by tethering to segregating chromosomes via BRD463. Future studies may address 505 whether an ecDNA hub serves as a unit of inheritance or merely as a transient congregation. The 506 observation of ecDNA hubs also warrants further investigation into whether this unusual 3D 507 organization of DNA molecules impacts other cellular processes regulated by genome 508 organization, such as DNA repair and replication. 
The recognition that ecDNA hubs promote oncogene transcription may provide new 510 therapeutic opportunities. While chromosomal DNA amplicons are covalently linked on an HSR, 511 ecDNA hubs are held together by proteins. In the case of the colorectal COLO320-DM cell line, 512 we show that BET protein inhibition by JQ1 disaggregates ecDNA hubs and reduces ecDNA513 derived MYC expression. JQ1 preferentially inhibited MYC transcription from ecDNA+ cancer 514 cells and inhibited both MYC and FGFR2 in SNU16 cells with dual oncogene hubs. Given that 515 ecDNA hubs are associated with high transcription, the observation that ecDNA hub maintenance 516 depends on BRD4 and/or other DNA-binding proteins may present a unique vulnerability of 517 ecDNA-driven cancers. The specific protein that mediates ecDNA clustering may be cancer-type 518 specific, as it likely needs to bind strongly to the endogenous sequences of the ecDNA-amplified 519 regions. Future studies using a broad screening approach coupled with analysis of ecDNA hub 520 maintenance may identify proteins that mediate of ecDNA clustering in various cancer types and 521 will be highly informative for potential therapeutic efforts.

\section{Figures}


a

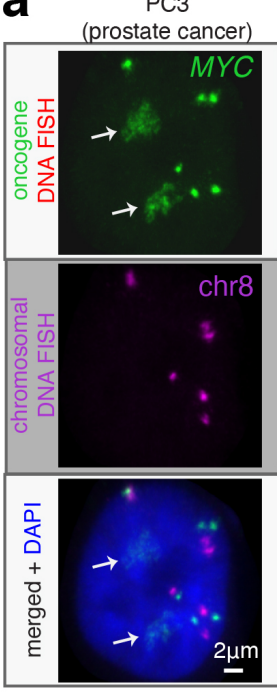

C
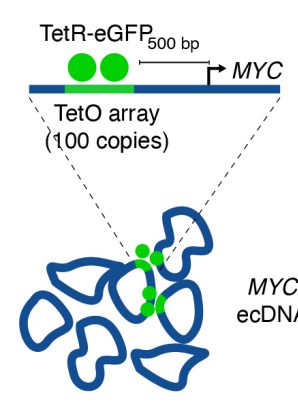

e

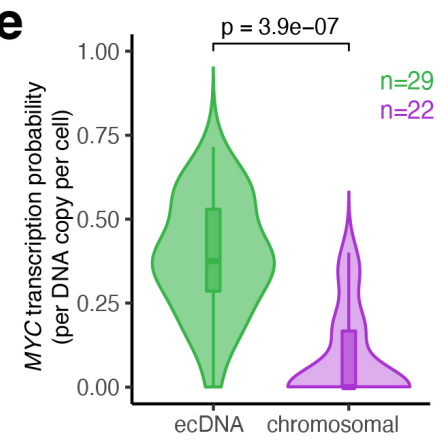

MYC
ecDNA

COLO320-DM
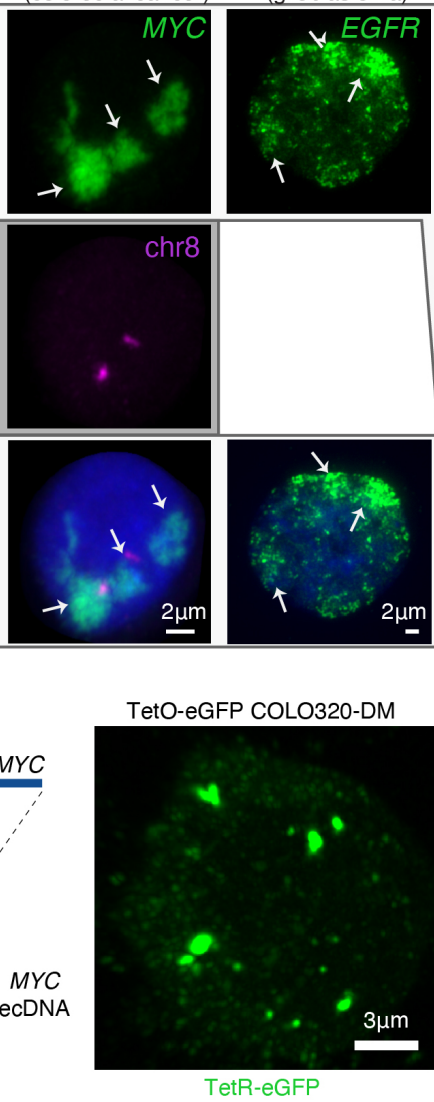

f

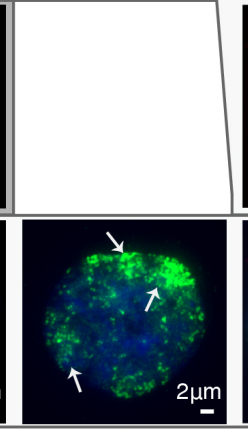

HK359
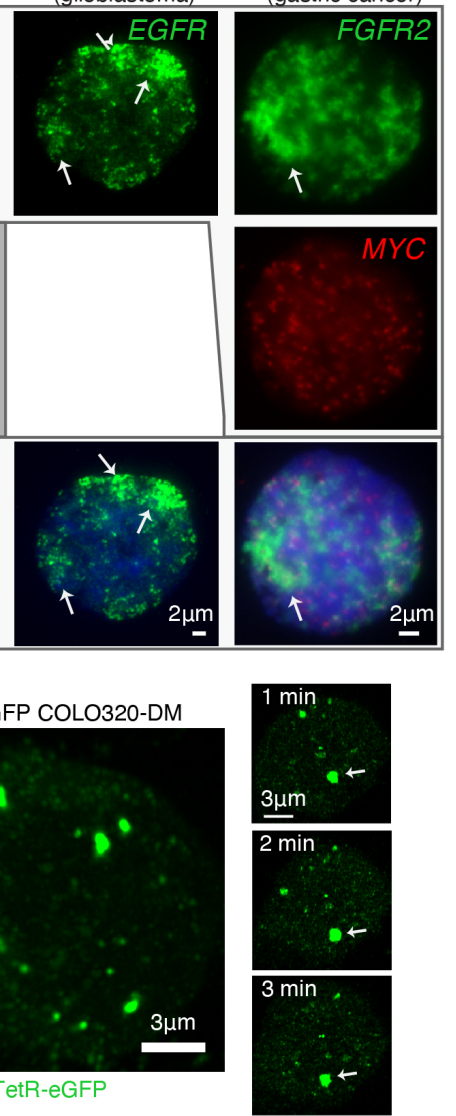
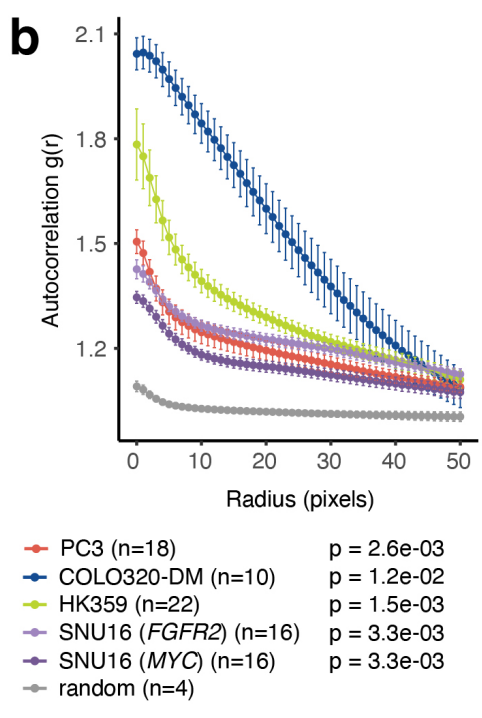

d
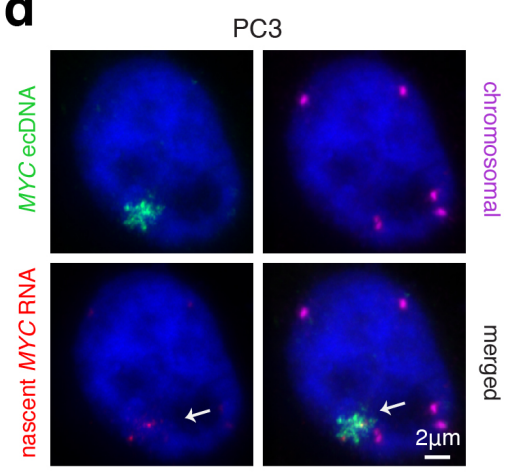

COLO320-DM (n=22)
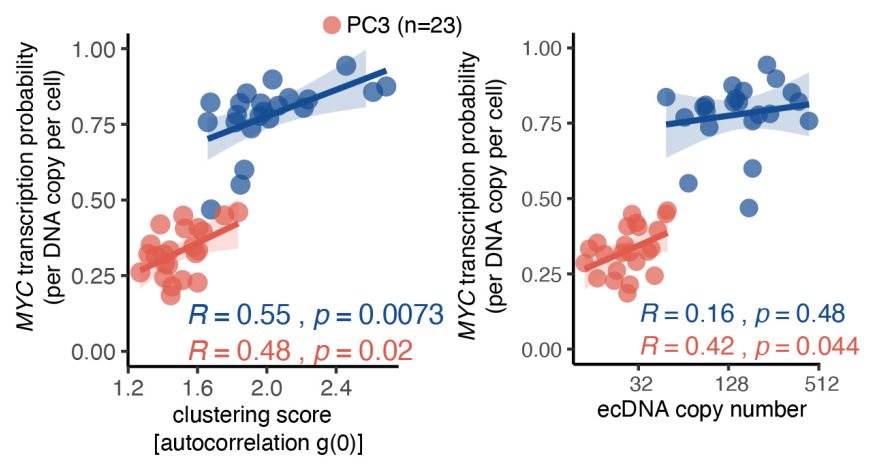

Figure 1. EcDNA imaging reveals ecDNA clustering and cooperative transcriptional bursting. (a) Representative FISH images showing ecDNA clustering during interphase in PC3 (MYC ecDNA, 1.5 Mb FISH probe), COLO320-DM (MYC ecDNA, $1.5 \mathrm{Mb}$ FISH probe), HK359 (EGFR ecDNA) and SNU16 (MYC and FGFR2 ecDNA, $200 \mathrm{~kb}$ MYC FISH probe) cells. For PC3 and COLO320-DM cells, a FISH probe targeting an adjacent chromosomal locus was also used. Scale bars, $2 \mu \mathrm{m}$. (b) Quantification of ecDNA clustering in interphase cells by autocorrelation $(\mathrm{g})$ at different pixel radii $(r)$. Data are represented as mean \pm SEM at each radial value. A pvalue comparing each cell line with random distribution is listed. P-values were calculated using the Wilcoxon test comparing the autocorrelation function values at radius $=0$. (c) Schematic for ecDNA imaging based on TetO array knock-in and labeling with TetReGFP (left). Representative images of TetR-eGFP signal in TetO-eGFP COLO320-DM cells at indicated timepoint during live cell imaging time course (right). Scale bars, $3 \mu \mathrm{m}$. (d) Representative image from combined DNA FISH for MYC ecDNA (100 kb probe) and adjacent chromosomal DNA with nascent MYC RNA FISH in PC3 cells. Scale bars, $2 \mu \mathrm{m}$. (e) Quantification of MYC transcription probability measured by nascent RNA FISH in (D) normalized to DNA copy number measured by DNA FISH for ecDNA and chromosomal loci (box center line, median; box limits, upper and lower quartiles; box whiskers, 1.58x interquartile range, here and throughout). (f) Scatter plots of ecDNA copy number (measured by DNA FISH) or ecDNA clustering score (measured by autocorrelation of ecDNA FISH signal at $r=0$ ) versus MYC transcription probability measured by nascent RNA FISH. Correlation coefficients calculated using Pearson's R. 


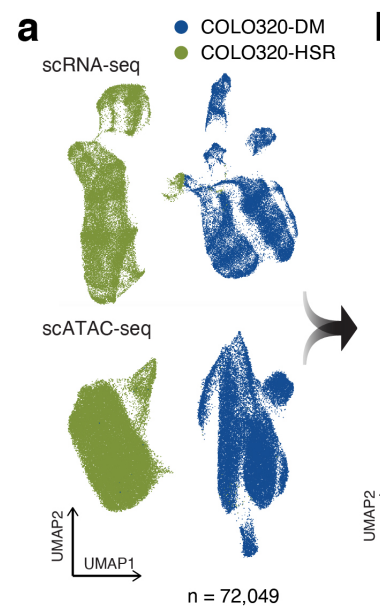

b

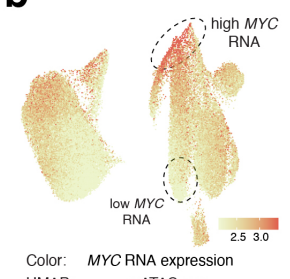

Color:
UMC RNA expression
UMAP:

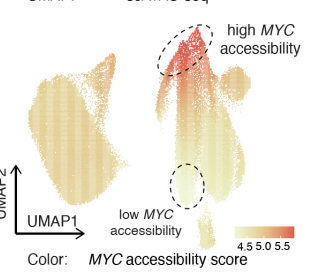

Color:
UMAP: $\quad$ accessibility s

C

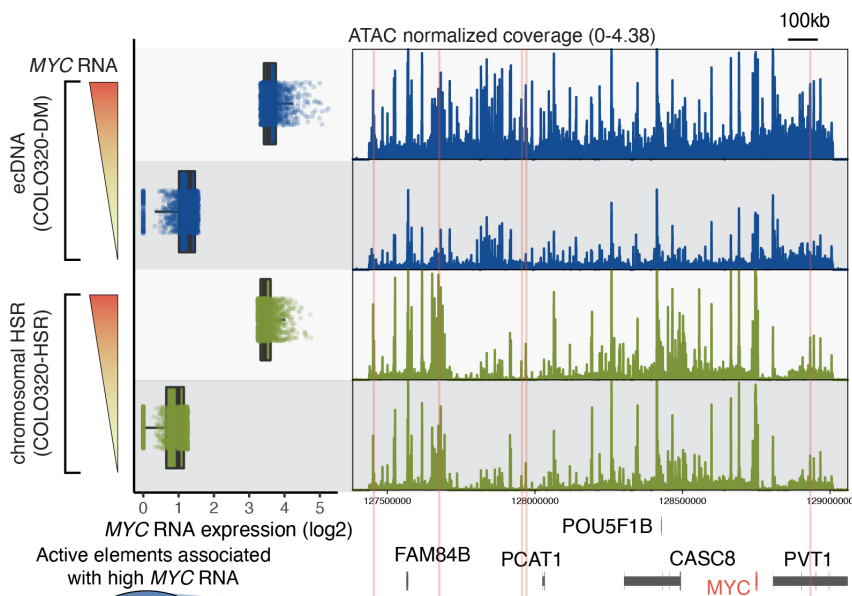

e
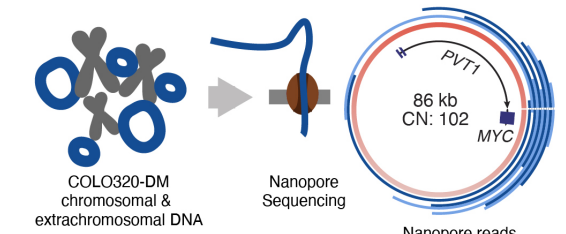
= Nanopore reads

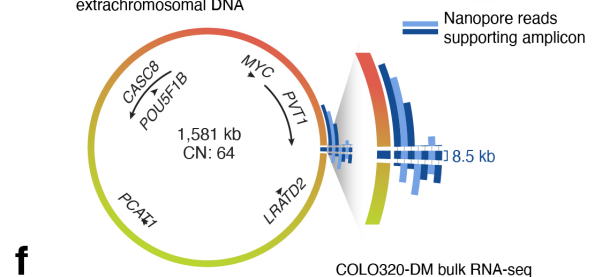

f
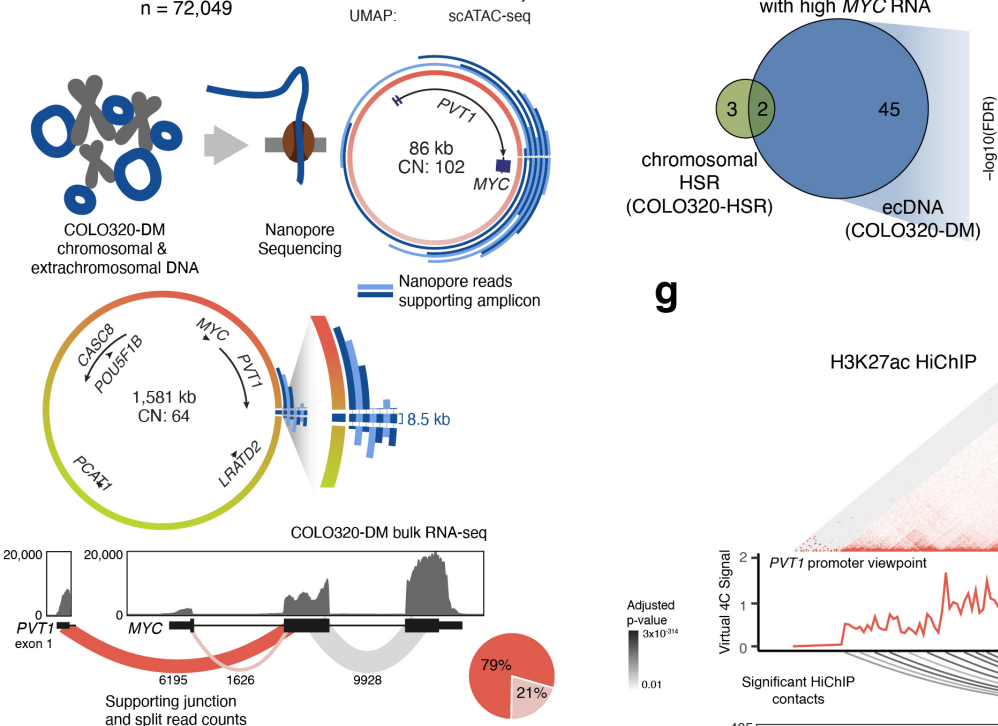

g
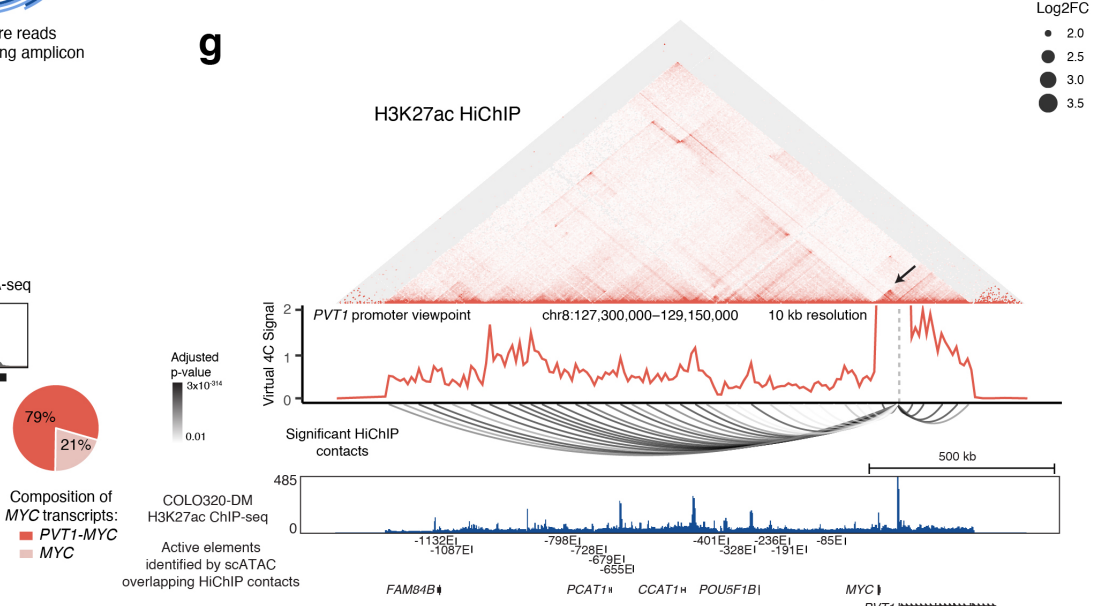

Figure 2. Genomic dissection identifies ecDNA enhancers associated with high MYC expression, ecDNA sequence heterogeneity and enhancer-promoter contacts. (a) COLO320-DM and COLO320-HSR cells profiled using single-cell paired RNA and ATAC-seq visualized with UMAP based on either the RNA or the ATAC-seq data. (b) Normalized MYC RNA expression levels were visualized on the ATAC-seq UMAP (top). An accessibility score for MYC was calculated using the ATAC-seq data and visualized on the UMAP with a color scale (bottom). (c) Cells were binned based on MYC RNA expression as outlined in Supplemental Figure $2 \mathrm{~b}$, and MYC expression levels of the top and bottom bins of COLO320-DM and COLO320-HSR are shown in a boxplot (left). The normalized ATAC-seq coverage for each bin is shown in the same order (right). (d) A venn diagram on the left shows number of variable elements identified on ecDNA amplicons in COLO320-DM compared to that identified on chromosomal HSRs in COLO320HSR. 45 variable elements were uniquely seen on ecDNA and 2 variable elements overlap with chromosomal HSRs. All 47 variable elements on ecDNA were plotted across the entire amplified region as a dot plot, in which the $\mathrm{x}$-axis represents genomic coordinates, the $y$-axis shows - $\log 10$ of the false discovery rate (FDR), and the size of each dot represents the log2 fold change (right). Five of these elements labelled and colored in red are the most significantly variable elements. They were named based on their relative position in kilobases in reference to the MYC transcriptional start site (TSS), with a negative number denoting an element 5 ' of MYC and a positive number denoting an element 3 ' of $M Y C$. The regions occupied by the marked elements were also highlighted in the coverage tracks correspondingly. (e) Schematic for nanopore sequencing of COLO320-DM cells (top left). Nanopore reads supporting MYC containing amplicon structures shown with alternating colors. (f) Bulk RNA sequencing coverage from COLO320-DM with exonexon junction spanning read counts shown (top). Pie chart quantifying relative abundance of full-length MYC and fusion PVT1-MYC transcripts using the read count supporting either junction (bottom). (g) H3K27ac HiChIP contact matrix (5 kb resolution, square root coverage normalized) in COLO320-DM cells centered on MYC locus (top). Arrow designates increased HiChIP signal at 86 kb PVT1MYC fusion amplicon. Virtual 4C plots from PVT1 promoter viewpoint with significant chromatin contacts identified by FitHiChIP shown below (middle). ATAC-seq signal track with active regulatory elements identified by scATAC-seq associated with high MYC expression that overlap loop anchors highlighted (bottom). Active elements named based on their relative position in kilobases in reference to the MYC transcriptional start site (TSS) as in panel c. 
bioRxiv preprint doi: https://doi.org/10.1101/2020.11.19.390278; this version posted November 20, 2020. The copyright holder for this preprint (which was not certified by peer review) is the author/funder. All rights reserved. No reuse allowed without permission.

Hung, Yost, Xie et al., (CHANG), p.19

a

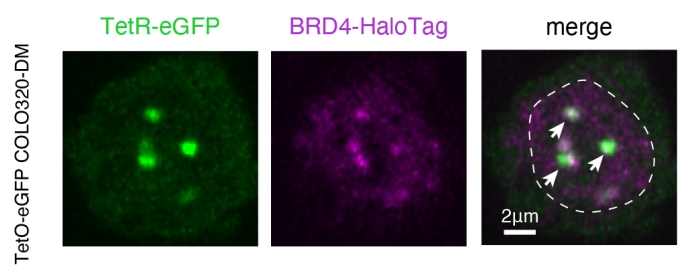

C
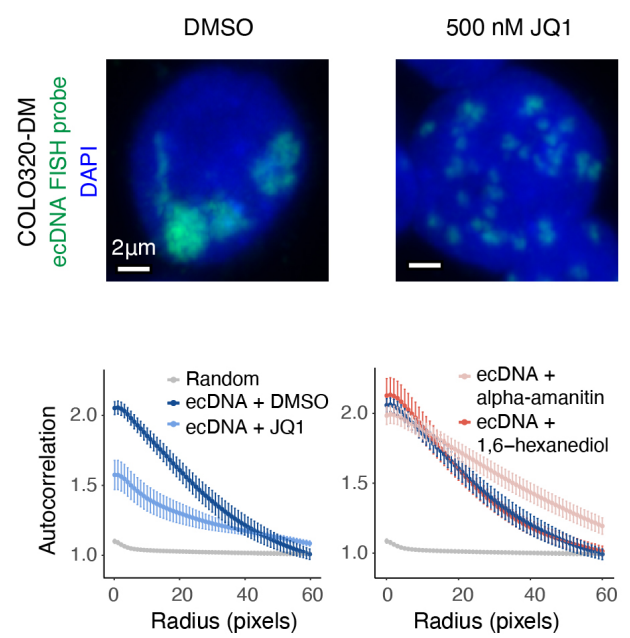

b

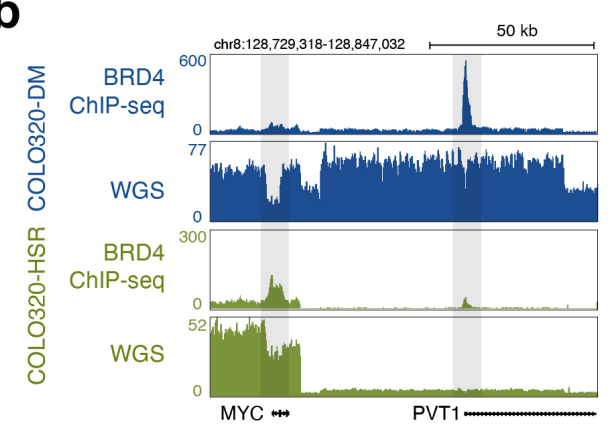

d

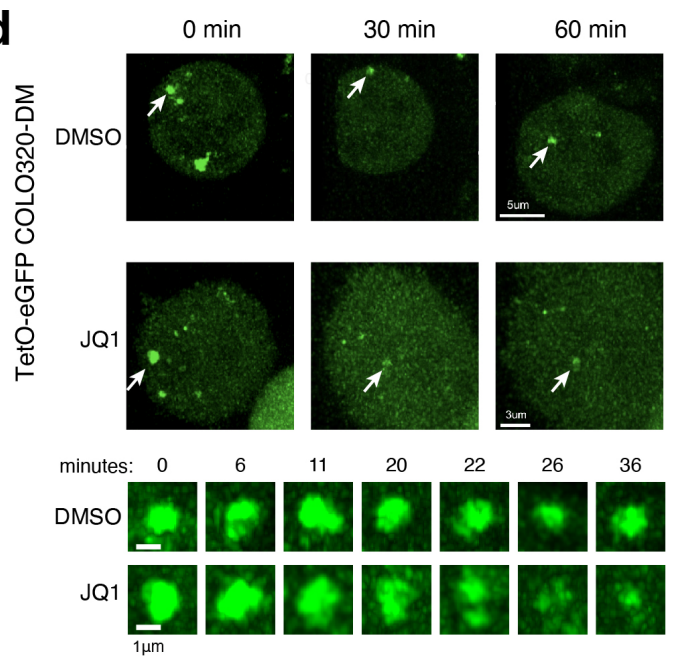

e

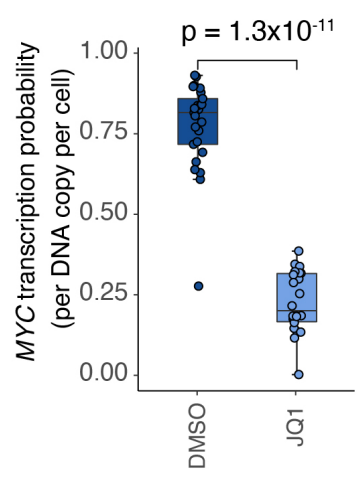

COLO320-DM f

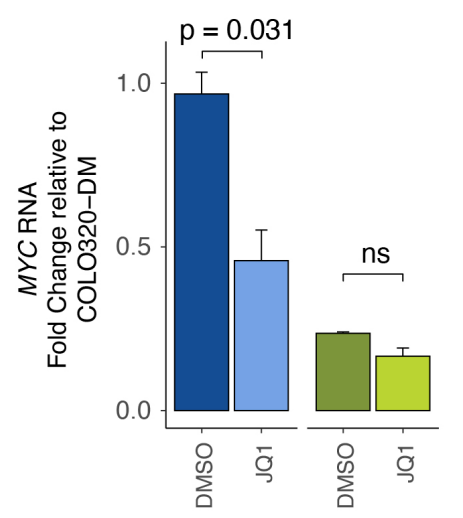

cocosest

Figure 3. BET proteins mediate ecDNA hub formation and transcription. (a) Representative image of eDNA labeled with TetReGFP in TetO-eGFP COLO320-DM cells overlaid with BRD4-HaloTag signal. Dashed line indicates nucleus boundary. (b) BRD4 ChIP-seq and whole genome sequencing coverage at MYC and PVT1 loci in COLO320-DM and COLO320-HSR cells. (c) Representative DNA FISH images for MYC ecDNA in interphase COLO320-DM cells treated with either DMSO or 500 nM JQ1 for 6 hours (top). Quantification of ecDNA clustering in interphase cells by autocorrelation ( $g$ ) at different pixel radii ( $r$ ) for COLO320-DM cells treated either with DMSO, $500 \mathrm{nM} \mathrm{JQ1,} \mathrm{1 \%} \mathrm{1,6-hexanediol,} \mathrm{or} 100 \mu \mathrm{g} / \mathrm{mL}$ alpha-amanitin for 6 hours. Error bars represent standard error between individual cells $(n=10)$ used for quantification (bottom). (d) Representative images of TetR-eGFP signal in TetO-eGFP COLO320-DM cells treated either with DMSO or $500 \mathrm{nM}$ JQ1 at indicated timepoint during live cell imaging time course. (e) Quantification of MYC transcription probability measured by nascent RNA FISH normalized to ecDNA copy number measured by DNA FISH for COLO320-DM cells treated either with DMSO or $500 \mathrm{nM}$ JQ1 for 6 hours $(\mathrm{n}=22-25)$. (f) Quantification of MYC RNA measured by RT-qPCR for COLO320-DM and COLO320-HSR cells treated either with DMSO or $500 \mathrm{nM}$ JQ1 for 6 hours $(\mathrm{n}=2)$. (g) Normalized cell viability for COLO320-DM and COLO320-HSR cells treated with a range of JQ1 concentrations for 48 hours normalized to cell viability for DMSO treated cells $(n=3)$. 
Hung, Yost, Xie et al., (CHANG), p.20

a

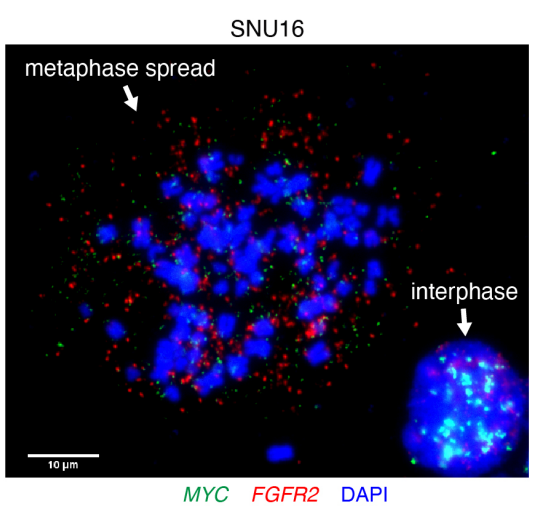

e
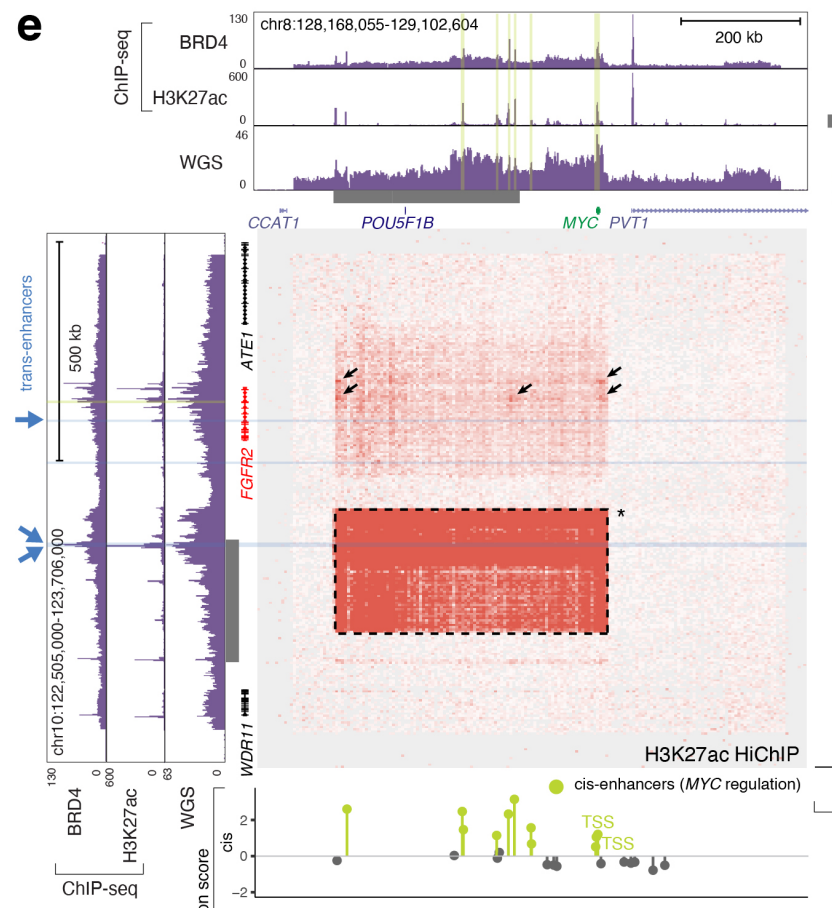

580

581 b

$M Y C$ ecDNA

FGFR2 eCDNA

MYC+FGFR2 ecDNA

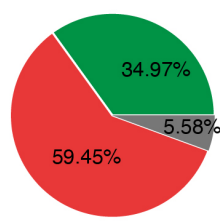

C

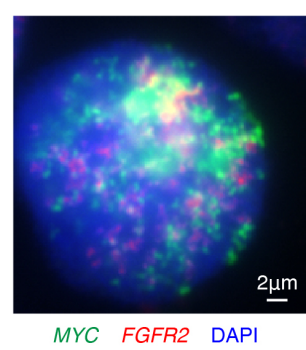

d

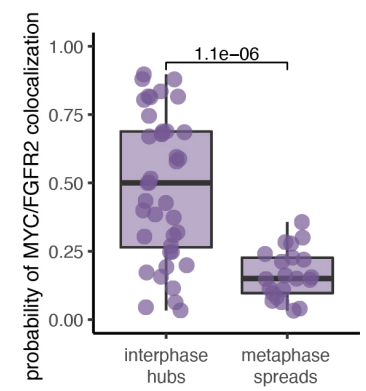

f

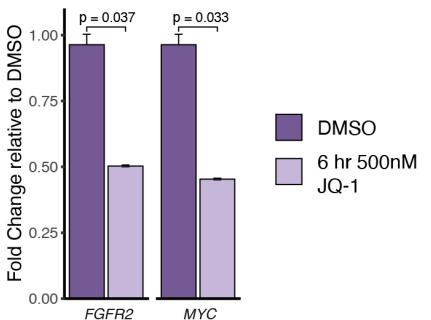

g

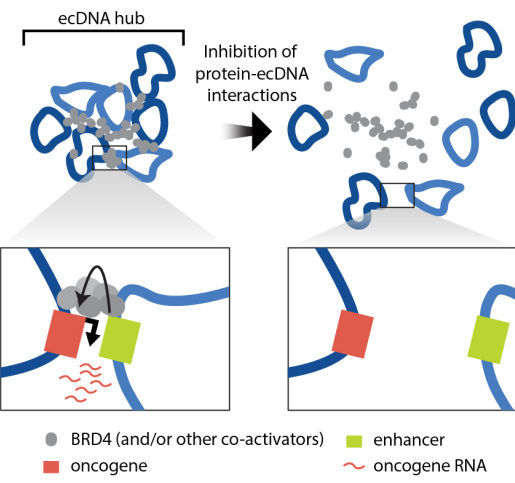

Figure 4. Intermolecular interaction among ecDNA molecules. (a) Representative FISH images showing extrachromosomal MYC and FGFR2 amplifications in metaphase spreads in SNU16 cells. (b) Quantification of MYC, FGFR2, and dual oncogene ecDNAs in SNU16 cells detected by DNA FISH in metaphase cells. (c) Representative DNA FISH images showing clustering of MYC and FGFR2 ecDNA during interphase in SNU16 cells. Scale bars, $2 \mu \mathrm{m}$. (d) Quantification of MYC and FGFR2 colocalization observed by DNA FISH in interphase and metaphase SNU16 cells. (e) H3K27ac HiChIP contact matrix (5 kb resolution, square root coverage normalized) in SNU16 cells between MYC and FGFR2 loci with 1D H3K27ac ChIP-seq, BRD4 ChIP-seq, and whole genome sequencing coverage tracks shown. Regions predicted to be covalently linked based on AmpliconArchitect analysis annotated in grey and region of potential cis interaction indicated by asterisk and dashed outline on HiChIP contact matrix. Area with focal contacts between MYC and FGFR2 loci highlighted. Lollipop plots (bottom and right) show the repressive effects of CRISPR interference on MYC or FGFR2 expression along the genomic track. Guides targeting putative enhancers that had a repressive effect on the oncogene in the same locus are marked as cis-enhancer targets (light green), whereas guides that repressed the oncogene in the other locus are marked as trans-enhancer targets (blue). Details of how the repression score was calculated can be found in Methods and Supplemental Figure 6c. Plots show data from two biological replicates. (f) Quantification of MYC and FGFR2 RNA measured by RTqPCR for SNU16 cells treated either with DMSO or 500 nM JQ1 for 6 hours. (g) A schematic diagram of the proposed ecDNA hub model for intermolecular cooperation. EcDNA hubs bring many heterogeneous ecDNA molecules into close proximity, allowing for interactions between enhancers and oncogenes on distinct molecules and promoting oncogene RNA transcription. The formation of these ecDNA hubs is mediated by BRD4 and/or other proteins that likely bind strongly to the endogenous sequences that are amplified on ecDNAs. Inhibition of protein-ecDNA interactions leads to dispersal of ecDNAs, which in turn reduces oncogene transcription. 
a
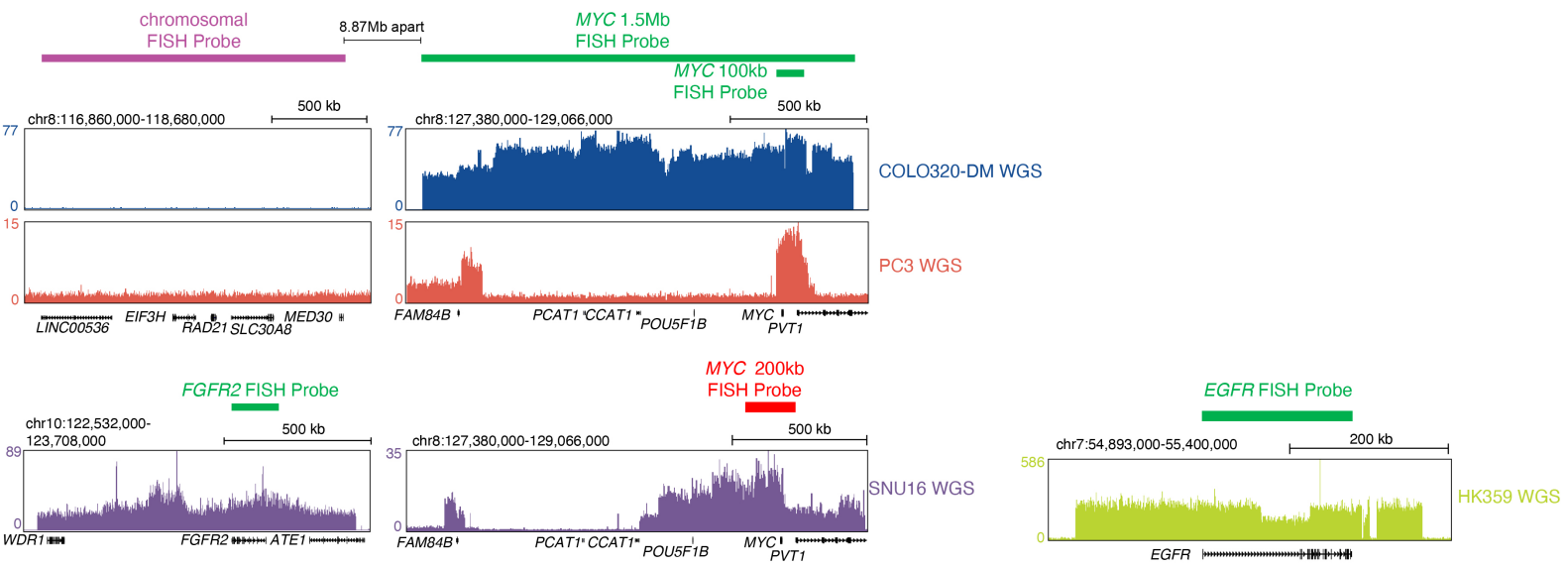

b

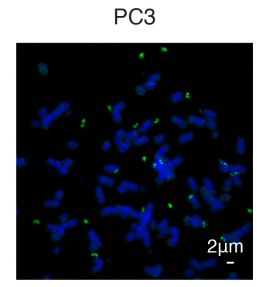

\section{COLO320-DM}

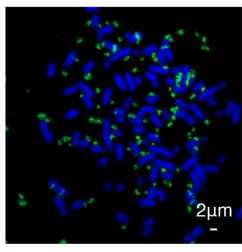

MYC DAPI

d

COLO320-DM

without TetO array integration

TetR-eGFP

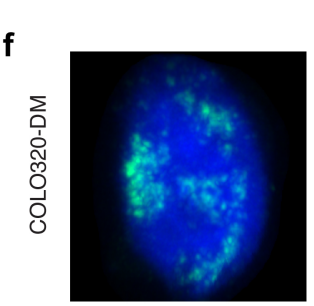

MYC ecDNA
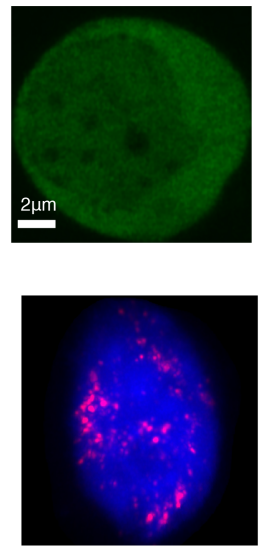

nascent MYC RNA

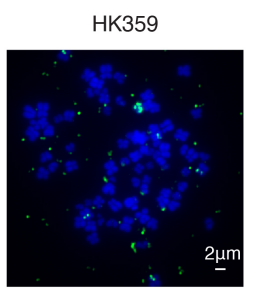

EGFR DAPI
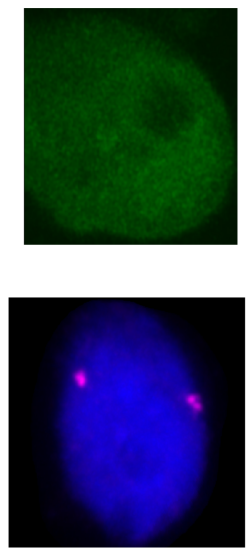

chromosomal

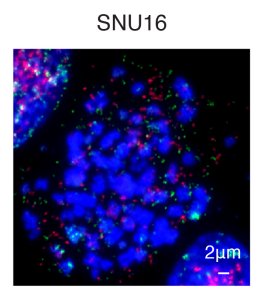

FGFR2 MYC DAPI
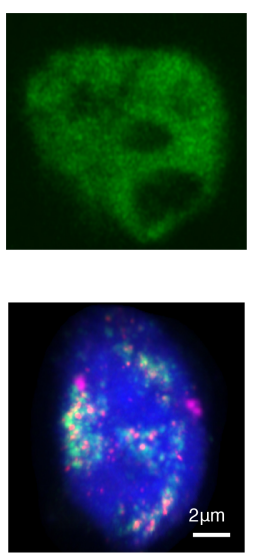

merged
C

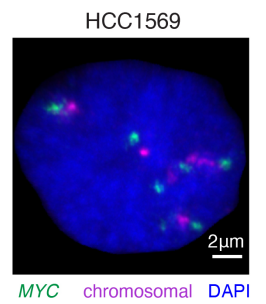

e
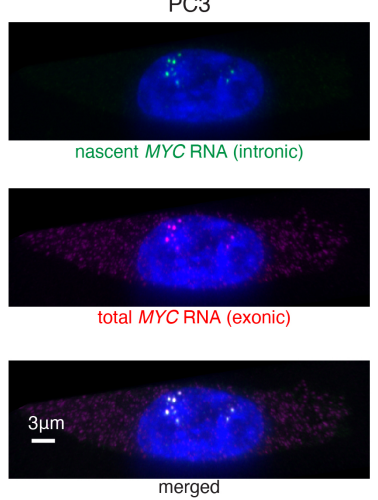

Supplemental Figure 1. EcDNA FISH probe design and validation of extrachromosomal FISH signal. (a) Whole genome sequencing signal tracks for PC3, COLO320-DM, HK359 and SNU16 cells with DNA FISH probe locations highlighted. For analysis of COLO320-DM and PC3 three FISH probes were used: a $1.5 \mathrm{Mb}$ Oligopaint FISH probe tiling the entire amplified locus surrounding MYC in COLO320-DM cells which was used for clustering analysis in Figure 1a,b; a $100 \mathrm{~kb}$ Oligopaint FISH probe at the MYC coding sequence used for ecDNA copy number quantification in Figure 1d,e,f; and a $1.5 \mathrm{Mb}$ Oligopaint FISH probe tiling an unamplified region located $8.87 \mathrm{Mb}$ upstream of the MYC locus on chromosome 8. For DNA FISH in SNU16 and HK359 cells, pre-designed FISH probes targeting either EGFR, FGFR2 or MYC were used. (b) Representative FISH images showing extrachromosomal amplifications in metaphase spreads for (MYC ecDNA, $200 \mathrm{~kb}$ probe), COLO320-DM (MYC ecDNA, $200 \mathrm{~kb}$ probe), HK359 (EGFR ecDNA) and SNU16 (MYC and FGFR2 ecDNA, $200 \mathrm{~kb}$ MYC probe) cells. Scale bars, $2 \mu \mathrm{m}$. (c) Representative DNA FISH images using chromosomal and $1.5 \mathrm{Mb}$ MYC probes in non-ecDNA amplified cell line HCC1569 during interphase. Scale bars, $2 \mu \mathrm{m}$. (d) Representative images of TetR-eGFP signal in COLO320-DM cells without TetO array integration. Scale bars, $2 \mu \mathrm{m}$. (e) Representative images of nascent MYC RNA FISH probe validation showing overlap of nascent MYC RNA (intronic) FISH probe and total MYC RNA (exonic) FISH probe in PC3 cells. Scale bars, $3 \mu \mathrm{m}$. (f) Representative images from combined DNA FISH for MYC ecDNA (100 kb probe) and adjacent chromosomal DNA with nascent MYC RNA FISH in COLO320-DM cells. Scale bars, $2 \mu \mathrm{m}$. 
bioRxiv preprint doi: https://doi.org/10.1101/2020.11.19.390278; this version posted November 20, 2020. The copyright holder for this preprint

(which was not certified by peer review) is the author/funder. All rights reserved. No reuse allowed without permission.

Hung, Yost, Xie et al., (CHANG), p.22

a

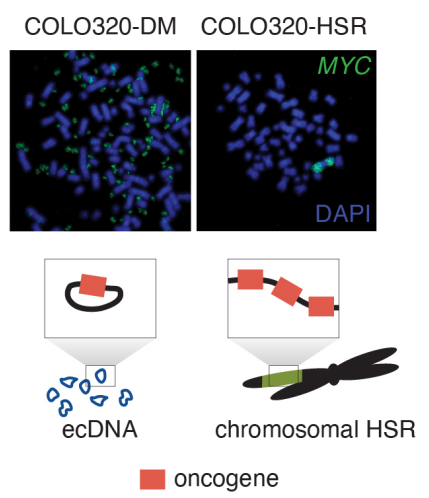

b

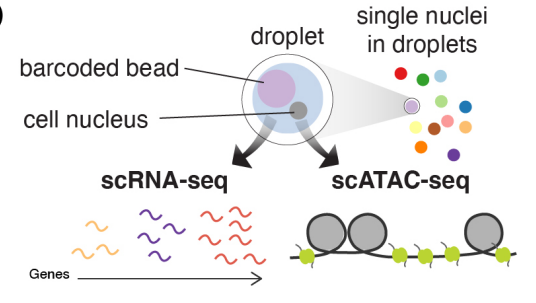

MYC expression chromatin accessibility

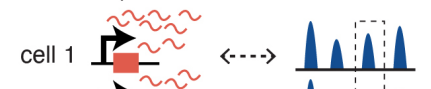

cell $2 \stackrel{\boldsymbol{P}}{2} \approx \ldots \rightarrow$

cell $n-1 \Perp \simeq \sim \cdots 11$

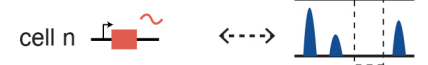

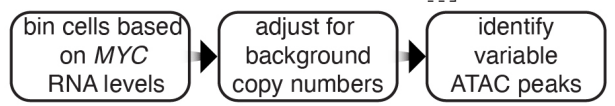

C
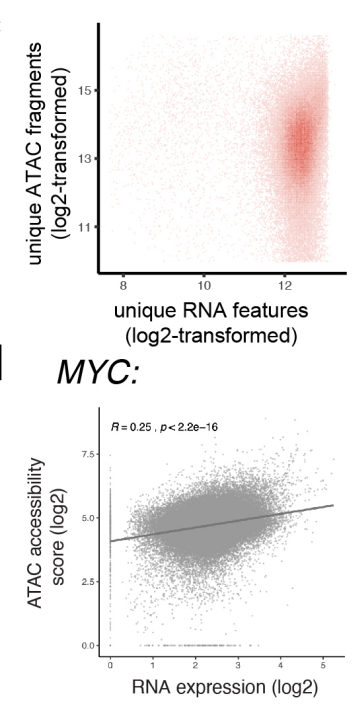

e

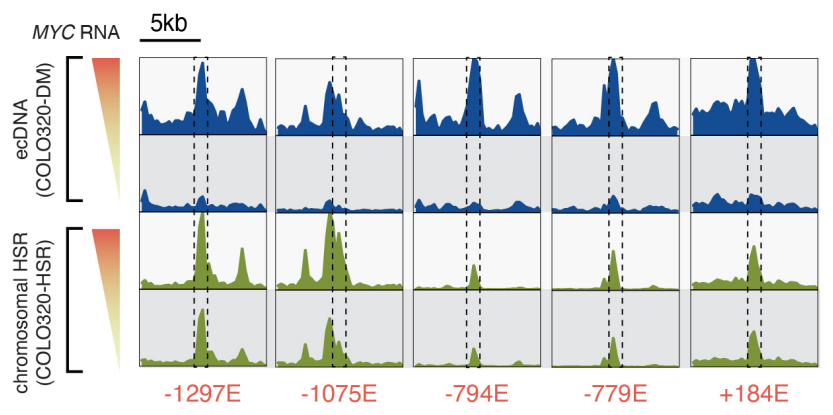

\section{g COLO320DM}

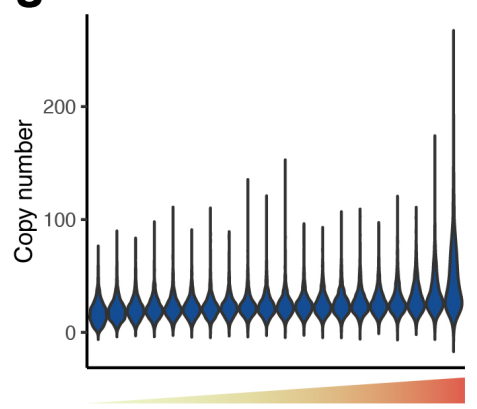

MYCRNA

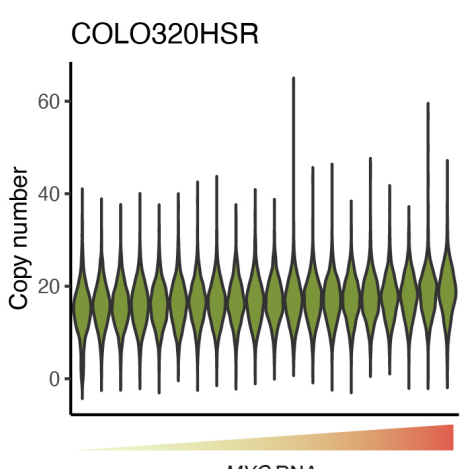

MYC RNA

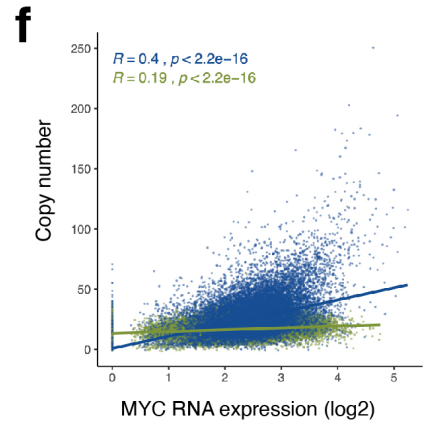

h

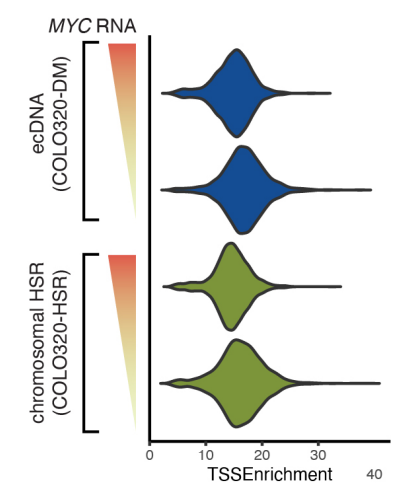

Supplemental Figure 2. Schematic and validation of paired single-cell RNA-seq and ATAC-seq analysis in COLO320-DM and COLO320-HSR. (a) Representative metaphase FISH images showing extrachromosomal MYC signals in COLO320-DM and chromosomal tandem MYC signals in COLO320-HSR. The schematic at the bottom illustrates oncogene amplification on circular ecDNA in COLO320-DM and chromosomal HSRs in COLO320-HSR. (b) Schematic showing the single-cell assay design and analysis. Droplets containing single nuclei and barcoded beads were generated on the 10X Genomics platform, and RNA and ATACseq reads were obtained from each single cell to simultaneously assay gene expression and chromatin accessibility. Cells were binned based on MYC RNA levels, the copy number of ecDNA or HSR amplicons for each cell was adjusted based on background ATAC signals, and variable ATAC-seq peaks were identified by differential marker testing between the top and the bottom bin. (c) Cells passing ATAC-seq and RNA-seq quality control filters are plotted based on unique ATAC-seq fragments and unique RNA features (both log2-transformed). (d) An accessibility score for MYC was calculated from the ATAC-seq data and plotted against normalized MYC expression from RNA-seq data, showing a positive correlation. (e) Zoom-ins of the ATAC-seq coverage of each of the five most significantly variable elements identified in Figure 2d. Dashed boxes mark the positions of these elements. (f) A scatter plot of estimated MYC amplicon copy numbers and normalized log2-transformed MYC expression of all individual cells showing positive correlations and a high level of copy number variability. (g) Estimated MYC amplicon copy number distributions of all RNA bins of COLO320-DM and COLO320-HSR. (h) A violin plot showing distributions of TSS enrichments in the high and low RNA bins of COLO320-DM and COLO320-HSR. 
bioRxiv preprint doi: https://doi.org/10.1101/2020.11.19.390278; this version posted November 20, 2020. The copyright holder for this preprint (which was not certified by peer review) is the author/funder. All rights reserved. No reuse allowed without permission.

Hung, Yost, Xie et al., (CHANG), p.23

a

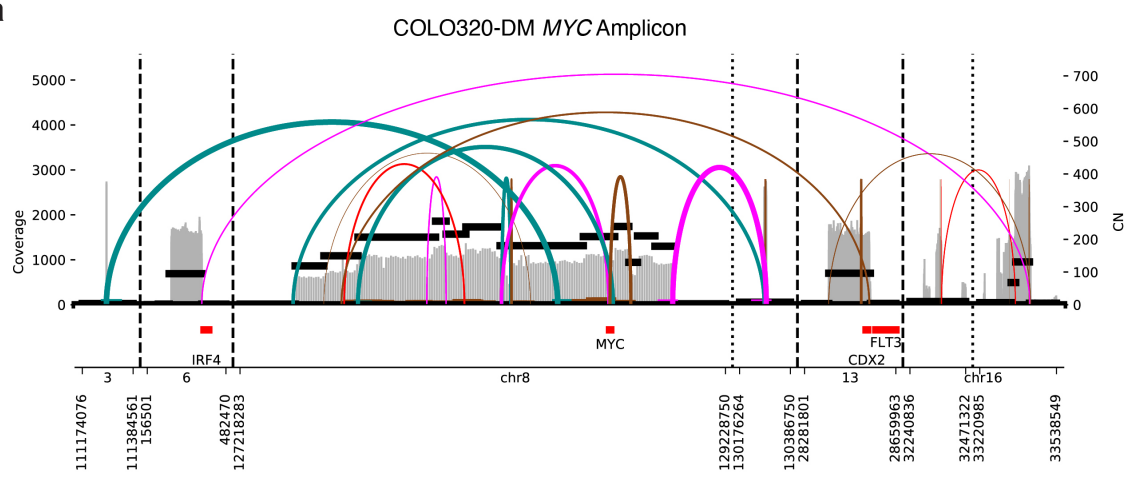

b
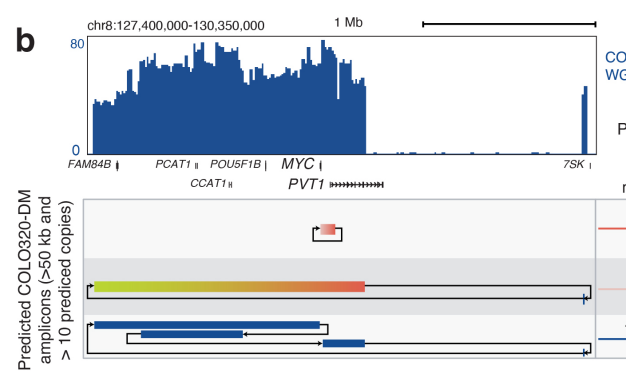

d

$7 S K$

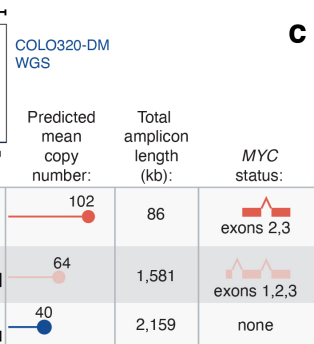

C

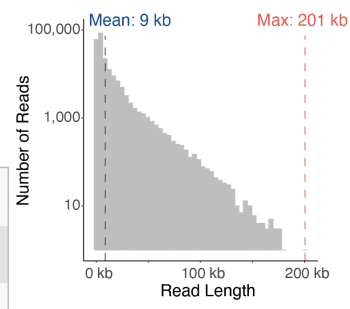

e

Transcriptional co-variation of
MYC and other amplified genes (scRNA-seq)
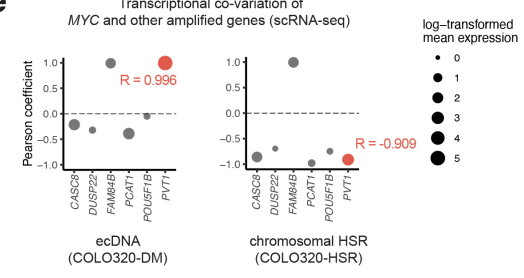

: 0

$1 \mathrm{Mb}$
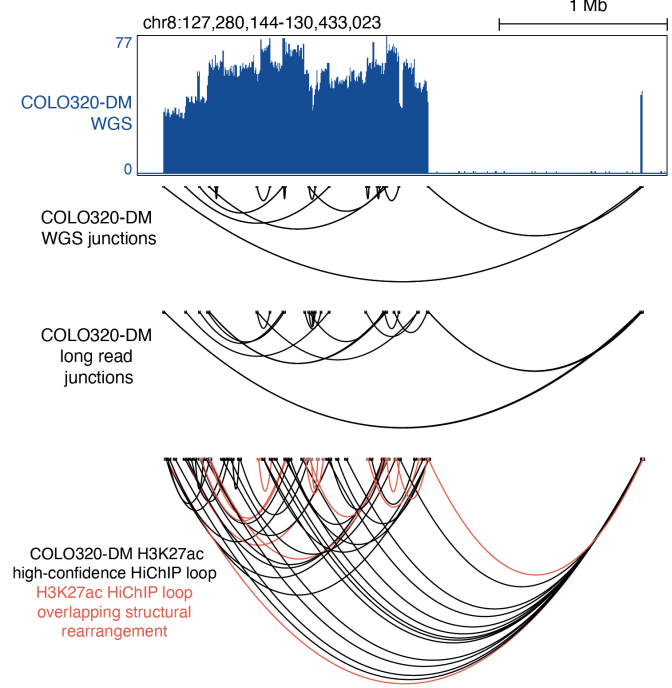

f

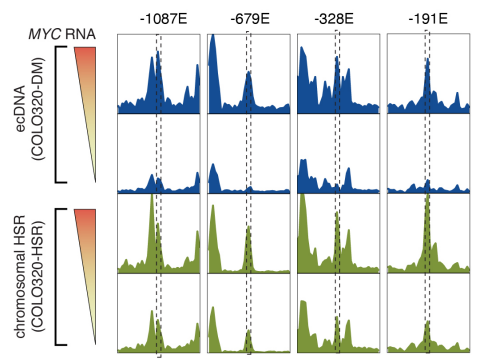

g

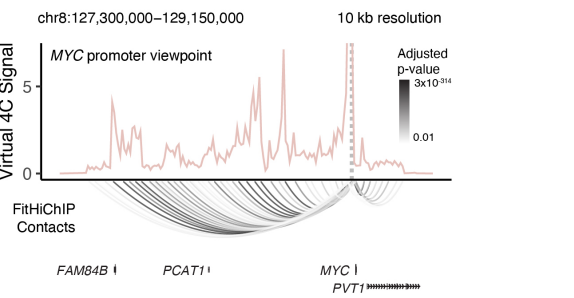

Supplemental Figure 3. ecDNA sequence heterogeneity and enhancer-promoter contacts. (a) Structural variant (SV) view of AmpliconArchitect (AA) reconstruction of the MYC amplicon in COLO320-DM cells. (b) Whole genome sequencing coverage from COLO320-DM and amplicon structures predicted by AmpliconArchitect, annotated with predicted mean copy number, total amplicon length, and MYC status. DNA segments shown as blocks with arrows designating junctions supported by whole genome sequencing. Amplicon structures with over $50 \mathrm{~kb}$ in total length, over 10 predicted copies, and with head-to-tail orientation characteristic of circular amplicons shown. (c) Distribution of read lengths from long-read nanopore sequencing of COLO320-DM. (d) Whole genome sequencing coverage from COLO320-DM (top). Junctions detected by whole genome sequencing, junctions detected by long-read nanopore sequencing, and H3K27ac HiChIP high-confidence loops identified by HICCUPS shown below, with HiChIP loops overlapping structural rearrangements highlighted. (e) Pearson correlation of transcription co-variation from scRNA-seq quantification of MYC and other amplified genes in COLO320-DM and COLO320-HSR cells. (f) Aggregate scATAC-seq signal tracks from top MYC RNA expression bin and bottom MYC RNA expression bin in COLO320-DM and COLO320-HSR cells, with differential peaks associated with high MYC RNA expression and overlapping HiChIP loop anchors highlighted. (g) COLO320-DM H3K27ac HiChIP virtual $4 \mathrm{C}$ plots from the MYC promoter viewpoint with FitHiChIP loops shown below, colored by adjusted $p$-value. 
bioRxiv preprint doi: https://doi.org/10.1101/2020.11.19.390278; this version posted November 20, 2020. The copyright holder for this preprint (which was not certified by peer review) is the author/funder. All rights reserved. No reuse allowed without permission.

Hung, Yost, Xie et al., (CHANG), p.24
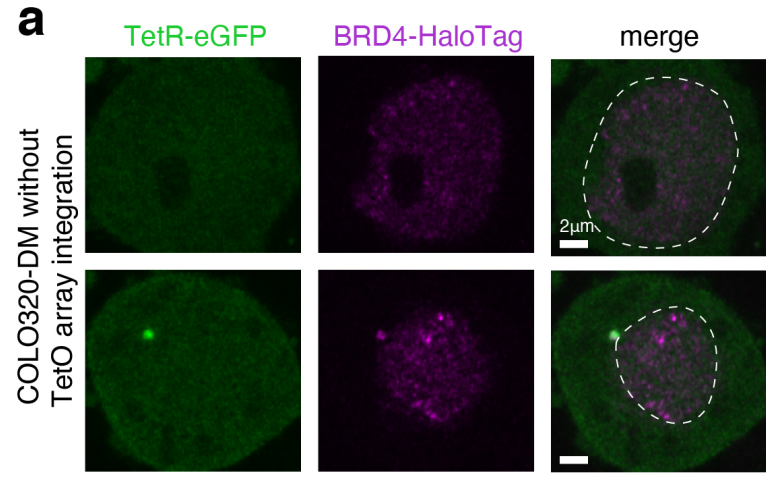

\section{C}
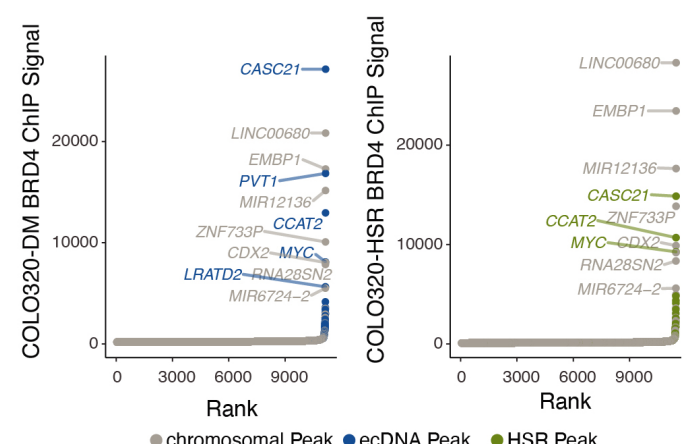

b

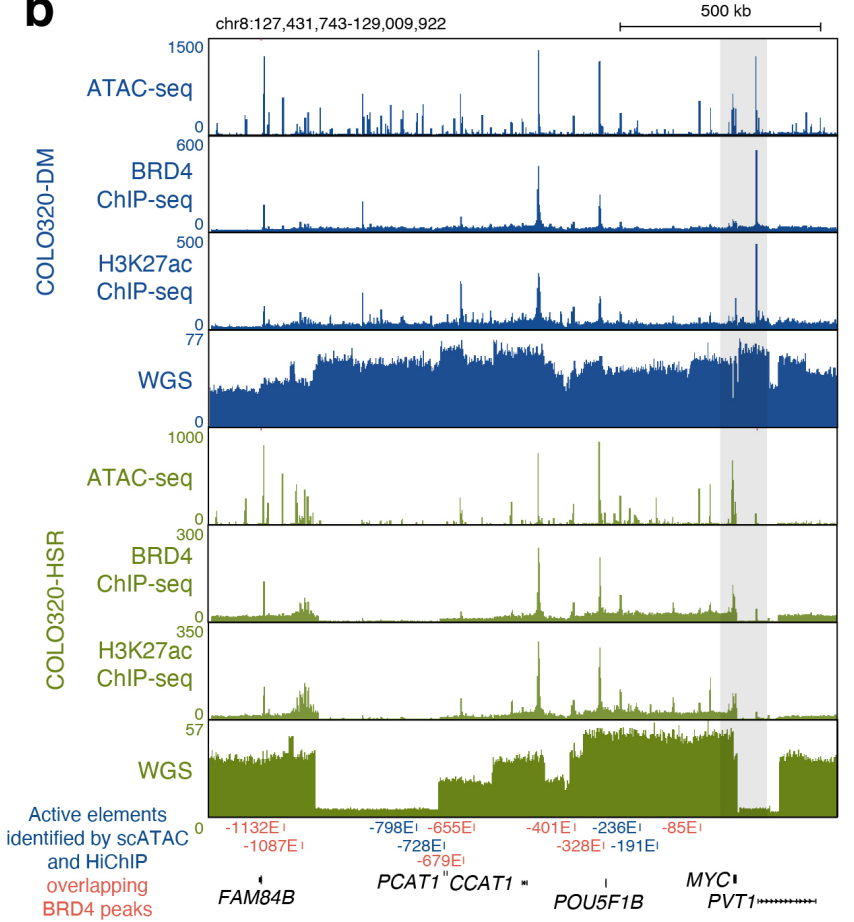
Supplemental Figure 4. ecDNA is enriched for BRD4 occupancy. (a) Representative image of TetR-eGFP signal in COLO320-
DM cells without TetO array integration overlaid with BRD4-HaloTag signal. Dashed line indicates nucleus boundary. We noted cytoplasmic TetR-eGFP signal in a subset of COLO320-DM cells without TetO array integration during co-detection of BRD4-HaloTag (bottom) but these signals were located outside of the nuclear boundary and did not colocalize with BRD4-HaloTag. (b) ATAC-seq, BRD4 ChIP-seq, H3K27ac ChIP-seq and whole genome sequencing coverage tracks for COLO320-DM and COLO320-HSR cells at amplified MYC locus. Active regulatory elements identified in Figure 3D shown below with elements overlapping BRD4 ChIP-seq peaks highlighted. (c) Ranked BRD4 ChIP-seq signal for COLO320-DM and COLO320-HSR, which peaks included in either ecDNA or HSR amplifications highlighted. 

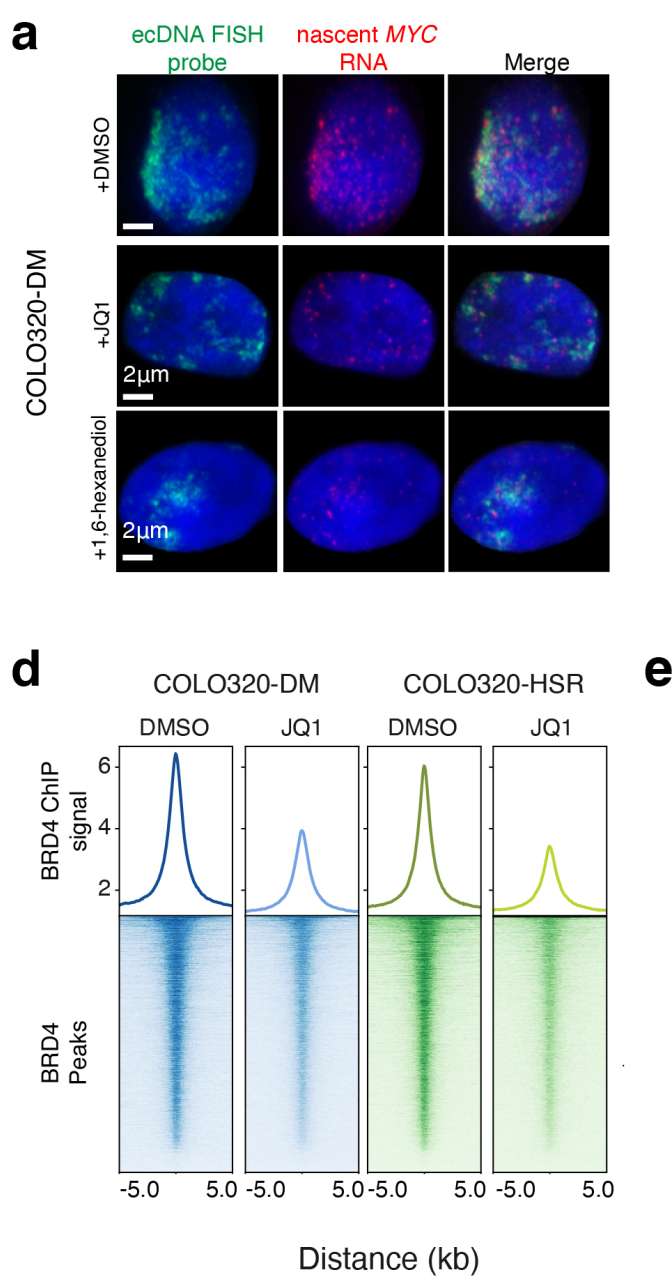

e
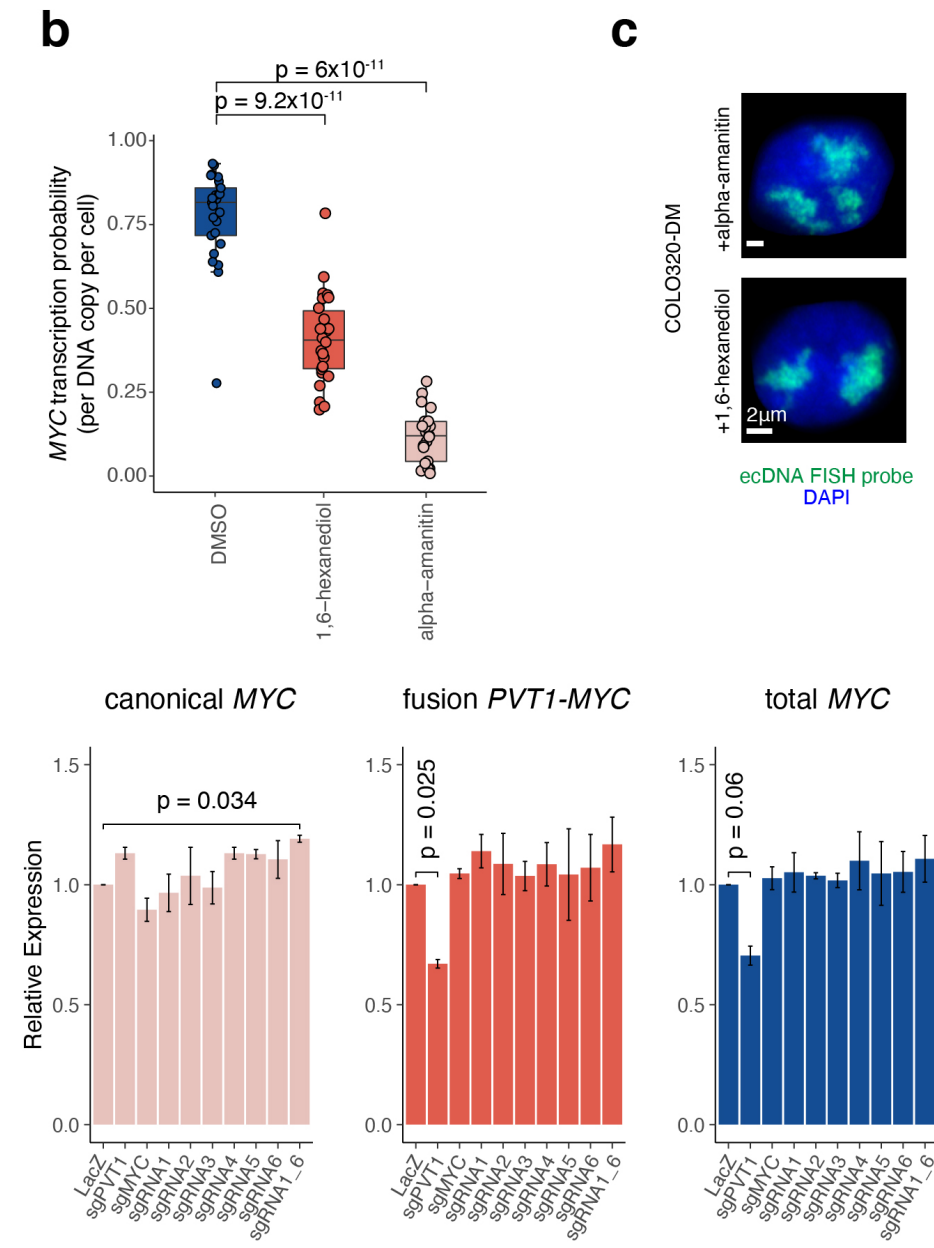

Distance $(\mathrm{kb})$

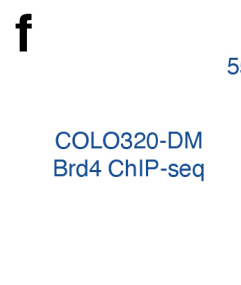

$$
\text { PCAT1H }
$$

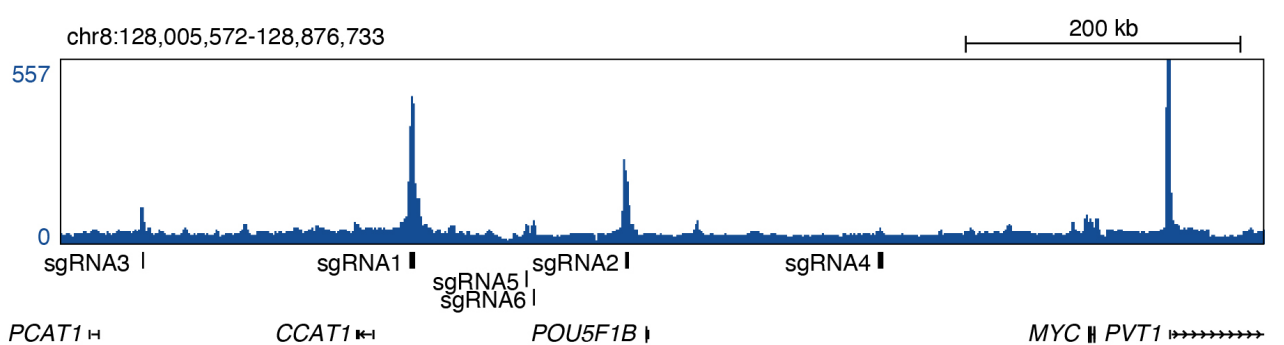

Supplemental Figure 5. ecDNA transcription following 1,6-hexanediol treatment and inhibition of individual enhancers. (a) Representative image from combined DNA FISH for MYC ecDNA with nascent MYC RNA FISH in COLO320-DM cells treated with DMSO, $500 \mathrm{nM}$ JQ1, or 1\% 1,6-hexanediol for 6 hours. (b) Quantification of MYC transcription probability measured by nascent RNA FISH normalized to ecDNA copy number measured by DNA FISH for COLO320-DM cells treated either with DMSO, 1\% 1,6hexanediol, or $100 \mu \mathrm{g} / \mathrm{mL}$ alpha-amanitin for 6 hours. (c) Representative DNA FISH images for MYC ecDNA in interphase COLO320DM cells treated with either $1 \%$ 1,6-hexanediol or $100 \mu \mathrm{g} / \mathrm{mL}$ alpha-amanitin for 6 hours. (d) Averaged BRD4 ChIP-seq signal (top) and heatmap of BRD4 ChIP-seq signal (bottom) over all BRD4 peaks for COLO320-DM and COLO320-HSR cells treated either with DMSO or $500 \mathrm{nM}$ JQ1 for 6 hours. (e) Relative RNA expression measured by RT-qPCR for indicated transcripts in COLO320-DM cells stably expressing dCas9-KRAB and indicated sgRNAs. Canonical MYC was amplified with primers MYC_exon1_fw and MYC_exon2_rv; fusion PVT1-MYC was amplified with PVT1_exon1_fw and MYC_exon2_rv; total MYC was amplified with total_MYC_exon2_fw and total_MYC_exon2_rv. All primer sequēnces are in Supplemental Täble 1. (f) BRD4 ChIP-seq coverage 
bioRxiv preprint doi: https://doi.org/10.1101/2020.11.19.390278; this version posted November 20, 2020. The copyright holder for this preprint (which was not certified by peer review) is the author/funder. All rights reserved. No reuse allowed without permission.

Hung, Yost, Xie et al., (CHANG), p.26

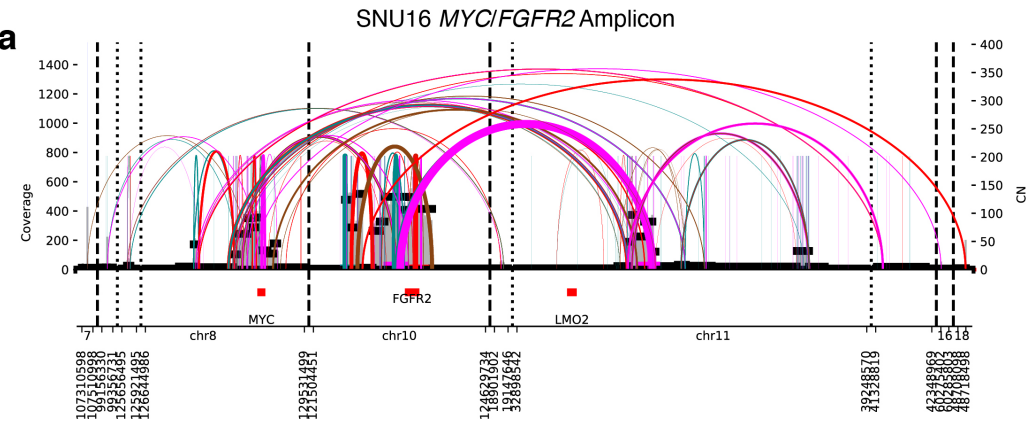

b

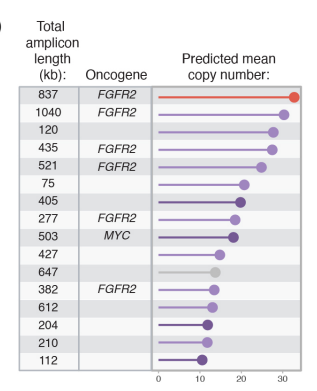

- chrro c chr11

chr8 chrs \& chr10
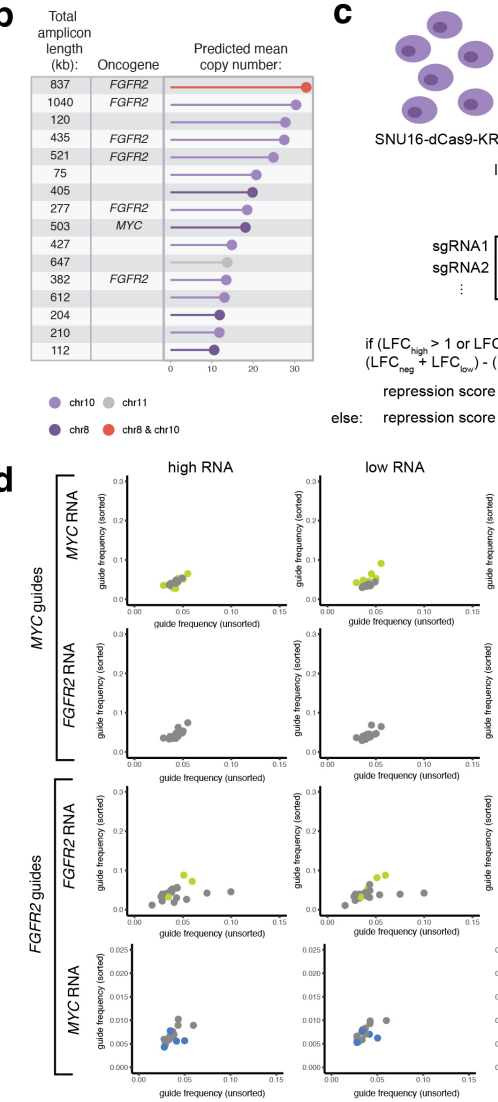

SNU16-dCas9-KRAB

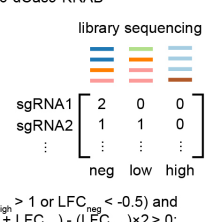

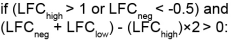

repression score $=0$

else: $\quad$ repression score $=\left(\mathrm{LFC}_{\text {neg }}+\mathrm{LFC}_{\text {low }}\right)-\left(\mathrm{LFC}_{\text {nigh }}\right) \times 2$
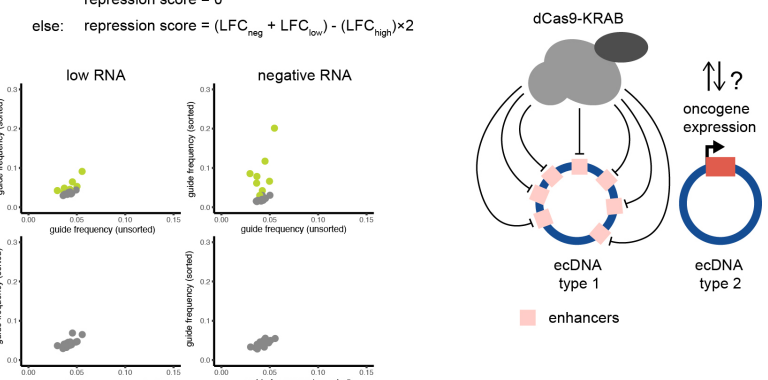

Supplemental Figure 6. Structural rearrangements of dual oncogene ecDNAs and CRISPR interference of their putative enhancers. (a) Structural variant (SV) view of AmpliconArchitect (AA) reconstruction of the MYCIFGFR2 amplicon in SNU16 cells. (b) Predicted amplicon length, oncogene content and mean copy number of MYC/FGFR2 amplicon structures in SNU16 cells. Amplicon structures with over $50 \mathrm{~kb}$ in total length and over 10 predicted copies shown. (c) A schematic of CRISPR interference experiments perturbing potentially trans-acting enhancers in SNU16 cells. Single guide RNAs (sgRNAs) were designed to target putative trans-enhancers on MYC-bearing ecDNAs (ecDNA type 1) based on chromatin accessibility, and expression of FGFR2 on distinct ecDNAs was measured (ecDNA type 2). A separate set of sgRNAs was designed to target putative trans-enhancers on FGFR2-bearing ecDNAs (ecDNA type 1), and expression of MYC was measured (ecDNA type 2) (bottom right). We performed these experiments in a pooled format. In brief, stable SNU16-dCas9-KRAB cells were generated and transduced with a lentiviral pool of sgRNAs targeting either the MYC-bearing ecDNAs or the FGFR2-bearing ecDNAs. Transduced cells were selected, oncogene RNA was labeled with FISH probes, FISH signals were amplified using PrimeFlow and assessed by flow cytometry. Cells with negative (neg), low or high RNA signals were sorted as different fractions, genomic DNA was extracted, and targeted libraries were prepared and sequenced. Relative abundances of sgRNAs were measured and a combined repression score was calculated by adding the log fold changes (LFCs) of each guide in the neg and low RNA fractions relative to unsorted cells, and subtracting from the LFC of each guide in the high RNA fraction. This repression score quantifies the degree to which each guide is enriched in the neg and low RNA fractions and depleted in the high RNA fraction. (d) Scatter plots showing relative frequencies of MYC ecDNA-targeting or FGFR2 ecDNA-targeting guides in cells with high, low or negative levels of MYC or FGFR2 RNA expression. Enhancers hits based on the combined repression scores are highlighted (cis in light green, trans in blue). We note that 8 of the FGFR2 guides had consistently higher frequencies in $M Y C$-sorted cells compared to unsorted cells. Because this enrichment is not differential between the high, low and negative RNA expressing cells, these guides do not fulfill our criteria outlined above for identifying guides with repressive effects on RNA expression and are therefore not categorized as hits. The $y$-axes in the bottom row was adjusted to exclude those guides. 
694 Supplemental Movie 1. Live cell imaging with untreated TetO-GFP COLO320-DM cells.

695 Snapshots of an untreated cell are shown over the course of 30 minutes. GFP labels TetO-knockin

696 MYC ecDNAs.

698 Supplemental Movie 2. Live cell imaging with DMSO-treated TetO-GFP COLO320-DM cells.

699 A control cell treated with DMSO was tracked over the course of 1 hour. GFP labels TetO-knockin

700 MYC ecDNAs.

702 Supplemental Movie 3. Live cell imaging with TetO-GFP COLO320-DM cells after JQ1

703 treatment. A cell treated with $500 \mathrm{nM}$ JQ1 was tracked over the course of 1 hour. GFP labels

704 TetO-knockin MYC ecDNAs. 
Hung, Yost, Xie et al., (CHANG), p.28

\section{METHODS}

\section{Cell Culture}

709 COLO320-DM, COLO320-HSR and HCC1569 cells were maintained in Roswell Park Memorial 710 Institute 1640 (RPMI; Life Technologies, Cat\# 11875-119) supplemented with 10\% fetal bovine 711 serum (FBS; Hyclone, Cat\# SH30396.03) and 1\% penicillin-streptomycin (pen-strep; Thermo 712 Fisher, Cat\# 15140-122). PC3 cells were maintained in Dulbecco's Modified Eagle Medium 713 (DMEM; Thermo Fisher, Cat\# 11995073) supplemented with 10\% FBS and 1\% pen-strep. HK359 714 cells were maintained in DMEM/Nutrient Mixture F-12 (DMEM/F12 1:1; Gibco, Cat\# 11320-082), 715 B-27 Supplement (Gibco, Cat\# 17504044), 1\% pen-strep, GlutaMAX (Gibco, Cat\# 35050061), 716 human epidermal growth factor (EGF, $20 \mathrm{ng} / \mathrm{ml}$; Sigma-Aldrich, E9644), human fibroblast growth 717 factor (FGF, 20 ng/ml; Peprotech) and Heparin (5 ug/ml; Sigma-Aldrich, Cat\# H3149-500KU). 718 SNU16 cells were maintained in DMEM/F12 supplemented with $10 \%$ FBS and $1 \%$ pen-strep. All 719 cells were cultured at $37^{\circ} \mathrm{C}$ with $5 \% \mathrm{CO}_{2}$.

\section{RT-qPCR}

722 RNA was extracted using RNeasy Plus mini Kit (QIAGEN 74136). Purified RNA was quantified 723 by Nanodrop (Thermo Fisher). For RT-qPCR, 50 ng of RNA, 1X Brilliant II qRT-PCR mastermix 724 with $1 \mathrm{uL}$ RT/RNase block (Agilent 600825), and $200 \mathrm{nM}$ forward and reverse primer were used. 725 Each Ct value was measured using Lightcycler 480 (Roche) and each mean dCt was averaged 726 from duplicate qRT-PCR reaction. Relative MYC RNA level (RT-qPCR primers MYC_exon3_fw 727 and MYC_exon3_rv) was calculated by ddCt method compared to 18S and GAPDH controls (RT728 qPCR primers GAPDH_fw, GAPDH_rv, 18S_fw, 18S_rv). The mean dCt value of each replicate 729 was used to calculate $p$ value using a Student's t-test. Primer sequences are listed in 730 Supplemental Table 1.

\section{Cell Viability Assays}

733 Cells were plated in 96-well plates at 25,000 cells/well and incubated either with JQ1 (Sigma734 Aldrich SML1524) at the indicated concentrations or an equivalent volume of DMSO for 48 hours.

735 Cell viability was measured using the CellTiterGlo assay kit (Promega G7572) in triplicate with 736 luminescence measured on SpectraMax M5 plate reader with an integration time of 1 second per 737 well. Luminescence was normalized to the DMSO treated controls and $p$ values calculated using 738 a Student's t-test. 
Hung, Yost, Xie et al., (CHANG), p.29

\section{Lentivirus production}

741 Lentiviruses were produced as previously described ${ }^{26}$. Briefly, 4 million HEK293Ts per $10 \mathrm{~cm}$

742 plate were plated the evening before transfection. Helper plasmids, pMD2.G and psPAX2, were

743 transfected along with the vector plasmid using Lipofectamine 3000 (Thermo Fisher, Cat\# L3000)

744 according to the manufacturer's instructions. Supernatants containing lentivirus were harvested

74548 hours later, filtered with a 0.45 um filter and concentrated using Lenti-X concentrator (Clontech,

746 Cat\#631232) and stored at $80^{\circ} \mathrm{C}$.

\section{Stable CRISPR cell line generation}

749 The pHR-SFFV-dCas9-BFP-KRAB (Addgene, Cat\# 46911) plasmid was modified to dCas9-BFP-

750 KRAB-2A-Blast as previously described ${ }^{26}$. Lentivirus was produced using the modified vector plasmid. Cells were transduced with lentivirus, incubated for 2 days, selected with $1 \mathrm{ug} / \mathrm{ml}$ blasticidin for 10-14 days, and BFP expression was analyzed by flow cytometry. To generate stable, monoclonal dCas9-KRAB cell lines, single BFP-positive cell clones were sorted into 96well plates and expanded. Vector expression was validated by flow cytometry.

\section{CRISPR interference}

757 sgRNAs were designed using the Broad Institute sgRNA designer online tool 758 (https://portals.broadinstitute.org/gpp/public/analysis-tools/sgrna-design). An additional guanine 759 was appended to each of the protospacers that do not start with a guanine. sgRNAs were cloned 760 into either mU6(modified)-sgRNA-Puromycin-mCherry or mU6(modified)-sgRNA-Puromycin761 EGFP previously generated ${ }^{26}$ and lentiviruses were produced. To evaluate the effects of CRISPR 762 interference on gene expression, cells were transduced with sgRNA lentiviruses, incubated for 2 763 days, selected with $0.5 \mathrm{ug} / \mathrm{ml}$ puromycin for 4 days, and BFP, GFP and/or mCherry expressions 764 were assessed by flow cytometry. Cells were harvested for RT-qPCR assays.

766 For the pooled experiments in SNU16, sgRNAs targeting bulk ATAC-seq peaks were designed, 767 cloned, pooled and lentiviruses were produced. SNU16-dCas9-KRAB cells were transduced with 768 the lentiviral guide pool, incubated for 2 days, selected with puromycin for 4 days, and RNA FISH 769 flow was performed for MYC and FGFR2 using the PrimeFlow ${ }^{\mathrm{TM}}$ RNA Assay Kit (Thermo Fisher) 770 following the manufacturer's protocol and corresponding probe sets (MYC: VA1-6000107-PF; 771 FGFR2: VA1-14785-PF). Cells were sorted by fluorescence-activated cell sorting (FACS), and 772 genomic DNA was extracted as previously described ${ }^{64}$. Libraries were prepared using 3 rounds 773 of PCR. The first round was performed using primers sgRNA_backbone_outer_fw and 
sgRNA_backbone_outer_rv to amplify guide sequences. The second round was a nested PCR with primers p5_mU6_0nt_stagger, p5_mU6_1nt_stagger, p5_mU6_2nt_stagger, 776 p5_mU6_3nt_stagger mixed at equimolar ratios and reverse primer p7adpt_spRNAl105nt_rev. 777 Finally, sequencing indices were attached in the third PCR using primers that anneal to the 778 adaptors and contain Illumina Truseq dual index primers. Initial amplification and nested PCR 779 primer sequences are listed in Supplemental Table 1. Amplified product sizes were validated on 780 a gel, and the products were purified using SPRlselect reagent kit (Beckman Coulter, Cat\# 781 B23318) at 1.2x sample volumes following the manufacturer's protocol. Libraries were sequenced 782 on an Illumina Miseq with paired-end 75 bp read lengths.

784 Relative abundances of sgRNAs were measured and compared using MAGeCK ${ }^{65}$. sgRNA counts 785 were obtained using the "mageck count" command, and differential enrichment analysis was 786 performed for each sorted cell fraction compared to unsorted cells using the "mageck test" command. Log fold changes (LFCs) relative to unsorted cells were obtained for each guide, and a combined repression score was calculated as $\left(\mathrm{LFC}_{\text {neg }}+\mathrm{LFC}_{\text {low }}\right)-\left(\mathrm{LFC}_{\text {high }}\right) \times 2$. In cases where $\mathrm{LFC}_{\text {high }}>1$ or $\mathrm{LFC}_{\text {neg }}<-0.5$ and the repression scores were above zero, we adjusted the repression scores to zero as the guides were considered to be enriched in cells with high expression and/or depleted in cells with low expression, i.e. they were non-repressive. All sgRNA sequences are listed in Supplemental Table 2.

\section{Metaphase chromosome spread}

795 Cells in metaphase were prepared by KaryoMAX (Gibco) treatment at $0.1 \mathrm{ug} / \mathrm{ml}$ for $3 \mathrm{hr}$. Single-

796 cell suspension was then collected and washed by PBS, and treated with $75 \mathrm{mM} \mathrm{KCl}$ for 15-30 797 min. Samples were then fixed by 3:1 methanol:glacial acetic acid, v/v and washed for an additional 798 three times with the fixative. Finally, the cell pellet resuspended in the fixative was dropped onto 799 a humidified slide.

\section{DNA FISH}

802 Slides containing fixed cells in interphase or metaphase were briefly equilibrated by $2 \mathrm{X}$ SSC, 803 followed by dehydration in $70 \%, 85 \%$, and $100 \%$ ethanol for 2 min each. FISH probes in 804 hybridization buffer (Empire Genomics) were added onto the slide, and the sample was covered 805 by a coverslip then denatured at $75^{\circ} \mathrm{C}$ for $1 \mathrm{~min}$ on a hotplate, and hybridized at $37^{\circ} \mathrm{C}$ overnight. 806 The coverslip was then removed, and the sample was washed one time by $0.4 \mathrm{X}$ SSC with $0.3 \%$ 807 IGEPAL, and two times by 2 X SSC with $0.1 \%$ IGEPAL, for 2 min each. DNA was stained with 
Hung, Yost, Xie et al., (CHANG), p.31

808 DAPI and washed with 2X SSC. Finally, the sample was mounted by mounting media (Molecular 809 Probes) before imaging.

811 The Oligopaint FISH probe libraries were constructed as described previously ${ }^{66}$. Each oligo 812 consists of a 40 nucleotide (nt) homology to the hg19 genome assemble designed from the 813 algorithm developed from the laboratory of Dr.Ting Wu (https://oligopaints.hms.harvard.edu/).

814 Each library subpool consists of a unique sets of primer pairs for orthogonal PCR amplification 815 and a $20 \mathrm{nt} \mathrm{T7}$ promoter sequence for in vitro transcription and a $20 \mathrm{nt}$ region for reverse 816 transcription. Individual Oligopaint probes were generated by PCR amplification, in vitro 817 transcription, and reverse transcription, in which ssDNA oligos conjugated with ATTO488 and 818 ATTO647 fluorophores were introduced during the reverse transcription step. The Oligopaint 819 covered genomic regions (hg19) used in this study are as follows: chr8:116967673-118566852 820 (hg19_COLO_nonecDNA_1.5Mbp), chr8:127435083-129017969 821 (hg19_COLO_ecDNA_1.5Mbp), chr8:128729248-128831223 (hg19_PC3_ecDNA1_100kb). A 822 ssDNA oligo pool was ordered and synthesized from Twist Bioscience (San Francisco, CA). 823 15mm \#1.5 round glass coverslips (Electron Microscopy Sciences) were pre-rinsed with 824 anhydrous ethanol for $5 \mathrm{~min}$, air dried, and coated with L-poly lysine solution (100ug/mL) for at 825 least 2 hours. Fully dissociated ColoDM320 or PC3 cells were seeded onto the coverslips and 826 recovered for at least 6 hours before experiments. Cells were fixed with $4 \%(\mathrm{v} / \mathrm{v})$ methanol free 827 paraformaldehyde diluted in $1 X$ PBS at room temperature for $10 \mathrm{~min}$. Then cells were washed $2 \mathrm{X}$ 828 with 1 XPBS and permeabilized in $0.5 \%$ Triton-X100 in 1 XPBS for 30min. After 2 X wash in 1 XPBS, 829 cells were treated with $0.1 \mathrm{M} \mathrm{HCl}$ for $5 \mathrm{~min}$, followed by $3 \mathrm{X}$ washes with $2 \mathrm{XSSC}$ and 30 min 830 incubation in 2X SSC $+0.1 \%$ Tween20 (2XSSCT) $+50 \%$ (v/v) formamide (EMD Millipore, 831 cat\#S4117). For each sample, we prepare 25ul hybridization mixture containing $2 X S S C T+50 \%$ 832 formamide $+10 \%$ Dextran sulfate (EMD Millipore, cat\#S4030) supplemented with $0.5 \mu 10 \mathrm{mg} / \mathrm{mL}$ 833 RNaseA (Thermo Fisher Scientific, cat\# 12091-021) $+0.5 \mu \mathrm{l} 10 \mathrm{mg} / \mathrm{mL}$ salmon sperm DNA 834 (Thermo Fisher Scientific, cat\# 15632011) and 20pmol probes with distinct fluorophores. The 835 probe mixture was thoroughly mixed by vortexing, and briefly microcentrifuged. The hybridization 836 mix was transferred directly onto the coverslip which was inverted facing a clean slide. The 837 coverslip was sealed onto the slide by adding a layer of rubber cement around the edges. Each 838 slide was denatured at $78^{\circ} \mathrm{C}$ for 4 min followed by transferring to a humidified hybridization 839 chamber and incubated at $42^{\circ} \mathrm{C}$ for 16 hours in a heated incubator. After hybridization, samples 840 were washed $2 X$ for 15 minutes in pre-warmed $2 X S S C T$ at $60^{\circ} \mathrm{C}$ and then were further incubated 841 at 2 XSSCT for $10 \mathrm{~min}$ at RT, at 0.2 XSSC for $10 \mathrm{~min}$ at RT, at 1 XPBS for 2 X5min with DNA 
Hung, Yost, Xie et al., (CHANG), p.32

842 counterstaining with DAPI. Then coverslips were mounted on slides with Prolong Diamond

843 Antifade Mountant (Thermo Fisher Scientific Cat\#P36961) for imaging acquisition.

\section{$845 \quad$ Nascent RNA FISH}

846 To quantify the MYC gene expression on the ecDNAs, we ordered the RNA FISH probes 847 conjugated with a Quasar 570 dye (Biosearch Technologies) targeting to the intronic region of 848 human (hg19) MYC gene for detection of nascent RNA transcript. We also ordered the RNA FISH 849 probes conjugated with a Quasar 670 dye targeting to the exonic region of human MYC gene for 850 detection of both mature and nascent RNA transcripts. For simultaneous detection of both ecDNA 851 and MYC transcription, 125nM RNA FISH probes was mixed with the DNA FISH probes (100kb 852 probe instead of the $1.5 \mathrm{Mbp}$ probe) together in the hybridization buffer without RNaseA and 853 incubated at $37^{\circ} \mathrm{C}$ overnight for $\sim 16$ hours. After hybridization, samples were washed $2 \mathrm{X}$ for 15 854 minutes in pre-warmed 2 XSSCT at $37^{\circ} \mathrm{C}$ and then were further incubated at 2 XSSCT for $10 \mathrm{~min}$ 855 at RT, at 0.2 XSSC for $10 \mathrm{~min}$ at RT, at 1 XPBS for $2 \times 5 \mathrm{~min}$ with DNA counterstaining with DAPI. 856 Then coverslips were mounted on slides with Prolong Diamond Antifade Mountant for imaging 857 acquisition.

\section{Microscopy}

860 DNA FISH images were acquired either with conventional fluorescence microscopy or confocal 861 microscopy. Conventional fluorescence microscopy was performed using an Olympus BX43 862 microscope, and images were acquired with a QiClick cooled camera. Confocal microscopy was 863 performed using a Leica SP8 microscope with lightning deconvolution (UCSD School of Medicine 864 Microscopy Core). Z-stacks were acquired over an average depth of approximately $8 \mu \mathrm{m}$, with 865 roughly $0.6 \mu \mathrm{m}$ step size.

DNA/RNA FISH images were acquired on the ZEISS LSM 880 Inverted Confocal microscope 868 attached with an Airyscan $32 \mathrm{GaAsP}$ PMT area detector. Before imaging, the beam position was 869 calibrated centering on the 32 detector array. Images were taken under the Airyscan SR mode 870 with a Plan Apochromat 63X/NA1.40 oil objective in a lens immersion medium having a refractive 871 index 1.515 at $30 \mathrm{oC}$. We used 405nm (Excitation wavelength) and 460nm (Emission wavelength) 872 for the DAPI channel, 488nm (Excitation wavelength) and 525nm (Emission wavelength) for the 873 ATTO488 channel, 561nm (Excitation wavelength) and 579nm (Emission wavelength) for the 874 Quasar570 channel and 633nm (Excitation wavelength) and 654nm (Emission wavelength) for 
Hung, Yost, Xie et al., (CHANG), p.33

875 the ATTO647 channel. Z-stacks were acquired with the optimal z sectioning thickness $~ 200 \mathrm{~nm}$,

876 followed by post-processing using the provided algorithm from ZEISS LSM880 platform.

\section{Generation of ecDNA-TetO array for live cell imaging}

879 sgRNA was designed by E-CRISP (http://www.e-crisp.org/E-CRISP/designcrispr.html) targeting $880 \sim 0.5 \mathrm{~kb}$ upstream of MYC transcription start site. The sgRNA sequence is listed in Supplemental

881 Table 2. The sgRNA was cloned into the modified pX330 (Addgene, Cat\# 42230) construct co882 expressing wild type SpCas9 and a PGK-Venus cassette. 500bp homology arms were PCR 883 amplified from COLO320-DM cells and cloned into a pUC19 donor vector together with 100 884 copies of TetO array and a blasticidin selection cassette. 2 ug of the donor vector and 1 ug of the 885 sgRNA vector were transfected into COLO320-DM cells by lipofectamine 3000 and blasticidin (10 $886 \mathrm{ug} / \mathrm{ml}$ ) selection was applied after 7 days. Individual clones were selected, genotyped by PCR 887 and verified by Sanger sequencing before being tested for imaging. To detect TetO array-labeled 888 ecDNA molecules, we transiently expressed TetR-eGFP as previously reported ${ }^{67}$ and performed 889 imaging experiments two days after transfection.

\section{Image Analysis}

892 To analyze the clustering of ecDNAs, we applied the autocorrelation function as described 893 previously ${ }^{68}$ in Matlab (2019). Specifically, the pair auto-correlation function $g(\vec{r})$ was calculated 894 by the fast Fourier transform (FFT) method described by the equations below.

$$
g(\vec{r})=\frac{F F T^{-1}\left(|F F T(I)|^{2}\right)}{\rho^{2} N(\vec{r})}
$$

$$
N(\vec{r})=F F T^{-1}\left(\mid\left. F F T(\text { Mask })\right|^{2}\right)
$$

$899 N(\vec{r})$ is the auto-correlation of a mask matrix that has the value of 1 inside the nucleus used for 900 normalization. The fast Fourier transform and its inverse ( $F F T$ and $F F T^{-1}$ ) were computed by fft2() 901 and ifft2() functions in Matlab, respectively. Autocorrelation functions were calculated first by 902 converting the Cartesian coordinates to polar coordinates by Matlab cart2pol() function, binning 903 by radius and by averaging within the assigned bins. For comparing auto-correlation with 904 transcription probability, the value of the auto-correlation function at radius of 0 pixels $(g(0))$ was 905 used to represent the degree of spatial clustering. The $g(0)$ values were also used for calculating 906 statistical significance among groups. 
Hung, Yost, Xie et al., (CHANG), p.34

908 To characterize the ecDNA shape and size, we employed the synthetic model—Surfaces object

909 from Imaris and applied a Gaussian filter ( $\sigma=1$ voxel in xy) before the downstream segmentation

910 and quantification. To measure the number of ecDNA or nascent transcripts, we localized the

911 voxels corresponding to the local maximum of identified DNA or RNA FISH signal using the Imaris

912 spots function module.

913

914 Colocalization analysis was performed using confocal images of both metaphase and interphase

915 nuclei from the same slides. Images were split into the two FISH colors, and background

916 fluorescence was removed manually for each channel. Colocalization for each nucleus was

917 quantified using the ImageJ-Colocalization Threshold program. Analysis was performed across

918 all z-stacks for each nucleus. Manders coefficient (fraction of MYC signal colocalized compared

919 to total MYC signal) was used to quantify colocalization.

\section{Whole Genome Sequencing}

922 Whole genome sequencing data from COLO320-DM, COLO320-HSR and PC3 cells were 923 generated by a previously published study ${ }^{1}$ and obtained from the NCBI Sequence Read Archive,

924 under BioProject accession PRJNA506071. Whole genome sequencing data from SNU16 cells 925 was generated by a previously published study ${ }^{69}$ and obtained from the NCBI Sequence Read 926 Archive, under BioProject accession PRJNA523380. Whole genome sequencing data from 927 HK359 cells was generated by a previously published study ${ }^{6}$ and obtained from the NCBI 928 Sequence Read Archive, under BioProject accession PRJNA338012.

$930 \quad$ Long Read Sequencing

931 Genomic DNA from COLO320-DM cells was extracted using a MagAttract HMW DNA Kit (Qiagen 932 67563) and prepared for long read sequencing using a Ligation Sequencing Kit (Oxford Nanopore 933 Technologies SQK-LSK109) according to the manufacturer's instructions. Sequencing was 934 performed on a MinION (Oxford Nanopore Technologies).

936 RNA-seq Library Preparation

937 COLO320-DM cells were transfected with Alt-R® S.p. Cas9 Nuclease V3 (IDT, Cat\# 1081058) 938 complexed with a non-targeting control sgRNA (Synthego) with a LacZ sequence following 939 Synthego's RNP transfection protocol using the Neon Transfection System (ThermoFisher, Cat\# 940 MPK5000). 500,000 to 1 million cells were harvested, and RNA was extracted using RNeasy Plus 941 mini Kit (QIAGEN 74136). Genomic DNA was removed from samples using the TURBO DNA- 
Hung, Yost, Xie et al., (CHANG), p.35

942 free kit (ThermoFisher, Cat\# AM1907), and RNA-seq libraries were prepared using the TruSeq

943 Stranded mRNA Library Prep (Illumina, Cat\# 20020595) following the manufacturer's protocol.

944 RNA-seq libraries were sequenced on an Illumina HiSeq 4000 with paired-end 75 bp read lengths.

946 ChIP-seq Library Preparation

947 Three million cells per replicate were fixed in $1 \%$ formaldehyde for 10 minutes at room

948 temperature with rotation and then quenched with $0.125 \mathrm{M}$ glycine for 10 minutes at room

949 temperature with rotation. Fixed cells were pelleted at $800 x g$ for 5 minutes at $4^{\circ} \mathrm{C}$ and washed

950 twice with cold PBS before storing at $-80^{\circ} \mathrm{C}$. Pellets were thawed and membrane lysis performed

951 in $5 \mathrm{~mL}$ LB1 (50 mM HEPES pH 8.0, $140 \mathrm{mM} \mathrm{NaCl}, 1 \mathrm{mM}$ EDTA, 10\% glycerol, 0.5\% NP-40,

$9520.25 \%$ Triton X-100, $1 \mathrm{mM} \mathrm{PMSF}$, Roche protease inhibitors 11836170001) for $10 \mathrm{~min}$ at $4^{\circ} \mathrm{C}$ with

953 rotation. Nuclei were pelleted at $1350 \times \mathrm{xg}$ for $5 \mathrm{~min}$ at $4^{\circ} \mathrm{C}$ and lysed in $5 \mathrm{~mL}$ LB2 (10 mM Tris-Cl

$954 \mathrm{pH}$ 8.0, 5 M, 200 mM NaCl, 1 mM EDTA, 0.5 mM EGTA, 1 mM PMSF, Roche protease inhibitors)

955 for 10 min at RT with rotation. Chromatin was pelleted at $1350 \mathrm{xg}$ for 5 min at $4^{\circ} \mathrm{C}$ and resuspended

956 in $1 \mathrm{~mL}$ of TE Buffer $+0.1 \%$ SDS before sonication on a Covaris E220. Samples were clarified by

957 spinning at $16,000 \times \mathrm{xg}$ for $10 \mathrm{~min}$ at $4^{\circ} \mathrm{C}$. Supernatant was transferred to a new tube and diluted

958 with 1 volume of IP Dilution Buffer (10 mM Tris pH 8.0, 1 mM EDTA, 200 mM NaCl, 1 mM EGTA.

$9590.2 \% \mathrm{Na}-\mathrm{DOC}, 1 \% \mathrm{Na}$-Laurylsarcosine, 2\% Triton X-100). Following addition of $20 \mathrm{ng}$ spike-in

960 chromatin (Active Motif 61686) and $2 \mu \mathrm{g}$ spike-in antibody (Active Motif 53083), $50 \mu \mathrm{L}$ of sheared

961 chromatin was reserved as input and ChIP performed overnight at $4^{\circ} \mathrm{C}$ with rotation with $7.5 \mu \mathrm{g}$

962 of antibody per IP: H3K27Ac (Abcam ab4729), BRD4 (Bethyl Laboratories A301-985A100).

$964100 \mu \mathrm{L}$ Protein G Dynabeads per ChIP were washed 3X in 0.5\% BSA in PBS and then bound to 965 antibody bound chromatin for 4 hours at $4^{\circ} \mathrm{C}$ with rotation. Antibody bound chromatin was washed 966 on a magnet 5X with RIPA Wash Buffer (50 mM HEPES pH 8.0, 500 mM LiCl, 1 mM EDTA, 1\% 967 NP-40, 0.7\% Na-Deoxycholate) and once with $1 \mathrm{~mL}$ TE Buffer (10 mM Tris-Cl pH 8.0, $1 \mathrm{mM}$ 968 EDTA) with $500 \mathrm{mM} \mathrm{NaCl}$. Washed beads were resuspended in $200 \mathrm{~mL}$ ChIP Elution Buffer (50 $969 \mathrm{mM}$ Tris- $\mathrm{Cl}$ pH 8.0, $10 \mathrm{mM}$ EDTA, 1\% SDS) and chromatin was eluted following incubation at $97065^{\circ} \mathrm{C}$ for $15 \mathrm{~min}$. Supernatant and input chromatin were removed to fresh tubes and reverse cross971 linked at $65^{\circ} \mathrm{C}$ overnight. Samples were diluted with $200 \mathrm{~mL}$ TE Buffer, treated with $0.2 \mathrm{mg} / \mathrm{mL}$ 972 RNase A (QIAGEN 19101) for 2 hours at $37^{\circ} \mathrm{C}$, then $0.2 \mathrm{mg} / \mathrm{mL}$ Proteinase $\mathrm{K}$ (New England 973 Biolabs P8107S) for 30 min at $55^{\circ} \mathrm{C}$. DNA was purified using the ChIP DNA Clean \& Concentrator 974 kit (Zymo Research D5205). ChIP sequencing libraries were prepared using the NEBNext Ultra 
Hung, Yost, Xie et al., (CHANG), p.36

975 II DNA Library Prep Kit for Illumina (New England Biolabs E7645S) with dual indexing (New

976 England Biolabs E7600S) following the manufacturer's instructions. ChIP-seq libraries were

977 sequenced on an Illumina HiSeq 4000 with paired-end 76 bp read lengths.

978

979 HiChIP Library Preparation

980 One to four million cells were fixed in 1\% formaldehyde in aliquots of one million cells each for 10 981 minutes at room temperature. HiChIP was performed as previously described ${ }^{40}$ using antibodies 982 against $\mathrm{H} 3 \mathrm{~K} 27 \mathrm{ac}$ (Abcam ab4729) with the following optimizations ${ }^{70}$ : SDS treatment at $62^{\circ} \mathrm{C}$ for $9835 \mathrm{~min}$; restriction digest with $\mathrm{Mbol}$ for $15 \mathrm{~min}$; instead of heat inactivation of Mbol restriction 984 enzyme, nuclei were washed twice with 1X restriction enzyme buffer; biotin fill-in reaction 985 incubation at $37^{\circ} \mathrm{C}$ for 15 minutes; ligation at room temperature for 2 hours. HiChIP libraries were 986 sequenced on an Illumina HiSeq 4000 with paired-end 76 bp read lengths. Single-Cell Paired RNA and ATAC-seq Library Preparation

989 Single-cell paired RNA and ATAC-seq libraries for COLO320-DM and COLO320-HSR were 990 generated on the 10x Chromium Single-Cell Multiome ATAC + Gene Expression platform 991 following the manufacturer's protocol and sequenced on an Illumina NovaSeq 6000.

\section{Long Read Sequencing Data Processing}

994 Bases were called from fast5 files using guppy (Oxford Nanopore Technologies, version 2.3.7).

995 Reads were then aligned using NGMLR ${ }^{71}$ (version 0.2.7) with the following parameters: -x ont -996 no-lowqualitysplit. Structural variants were called using Sniffles ${ }^{71}$ (version 1.0.11) using the 997 following parameters: -s 1 --report_BND --report_seq. Read information regarding mapping 998 locations and junctions present in individual reads stored in QS and QE read tags was extracted 999 using samtools and visualized in R.

\section{RNA-seq Data Processing}

1002 Paired-end reads were aligned to the hg19 genome using STAR-Fusion ${ }^{72}$ (version 1.6.0) and the 1003 genome build GRCh37_gencode_v19_CTAT_lib_Mar272019.plug-n-play. Number of reads 1004 supporting the PVT1-MYC fusion transcript were obtained from the "star1005 fusion.fusion_predictions.abridged.tsv" output file and the junction read counts and spanning 1006 fragment counts were combined. Reads supporting the canonical MYC exon 1-2 junction were 1007 obtained using the Gviz package in $R^{73}$ in a sashimi plot. 
Hung, Yost, Xie et al., (CHANG), p.37

\section{ChIP-seq Data Processing}

1010 Paired-end reads were aligned to the hg19 genome using Bowtie2 ${ }^{74}$ (version 2.3.4.1) with the -1011 very-sensitive option following adapter trimming with Trimmomatic ${ }^{75}$ (version 0.39). Reads with

1012 MAPQ values less than 10 were filtered using samtools and PCR duplicates removed using

1013 Picard's MarkDuplicates. MACS2 ${ }^{76}$ (version 2.1.1.20160309) was used for peak calling with the

1014 following parameters: macs2 callpeak -t chip_bed -c input_bed -n output_file -f BED -g hs -q

10150.01 --nomodel --shift 0. A reproducible peak set across biological replicates was defined using

1016 the IDR framework (version 2.0.4.2). Reproducible peaks from all samples were then merged to

1017 create a union peak set. ChIP-seq signal was converted to bigwig format for visualization using

1018 deepTools bamCoverage ${ }^{77}$ (version 3.3.1) with the following parameters: --bs 5 --smoothLength

1019105 --normalizeUsing CPM --scaleFactor 10. Enrichment of ChIP signal at peaks was

1020 performed using deepTools computeMatrix.

\section{HiChIP Data Processing}

$1023 \mathrm{HiChIP}$ data were processed as described previously ${ }^{40}$. Briefly, paired end reads were aligned to 1024 the hg19 genome using the HiC-Pro pipeline (version 2.11.0) ${ }^{78}$. Default settings were used to remove duplicate reads, assign reads to Mbol restriction fragments, filter for valid interactions, and generate binned interaction matrices. The Juicer pipeline's HiCCUPS tool and FitHiChIP were used to identify loops ${ }^{79,80}$. Filtered read pairs from the HiC-Pro pipeline were converted into .hic format files and input into HiCCUPS using default settings. Dangling end, self-circularized, and re-ligation read pairs were merged with valid read pairs to create a 1D signal bed file. FitHiChIP

1030 was used to identify "peak-to-all" interactions at $10 \mathrm{~kb}$ resolution using peaks called from the one1031 dimensional HiChIP data. A lower distance threshold of $20 \mathrm{~kb}$ was used. Bias correction was 1032 performed using coverage specific bias. HiChIP contact matrices stored in hic files were 1033 visualized in Juicebox using square root coverage normalization. Virtual 4C plots were generated 1034 from dumped matrices generated with Juicebox. The Juicebox tools dump command was used

1035 to extract the chromosome of interest from the hic file. The interaction profile of a 10-kb bin 1036 containing the anchor was then plotted in $\mathrm{R}$ following normalization by total number of valid read 1037 pairs and anchor bin signal.

\section{Single-Cell Paired RNA and ATAC-seq Data Processing}

1040 A custom reference package for hg19 was created using cellranger-arc mkref (10x Genomics, 1041 version 1.0.0). The single-cell paired RNA and ATAC-seq reads were aligned to the hg19 1042 reference genome using cellranger-arc count (10x Genomics, version 1.0.0). 
Hung, Yost, Xie et al., (CHANG), p.38

\section{Combined single-cell RNA and ATAC-seq analysis}

1045 Subsequent analyses on RNA were performed using Seurat ${ }^{81}$, and those on ATAC-seq were 1046 performed using ArchR ${ }^{31}$. Cells with more than 200 unique RNA features, less than $20 \%$ 1047 mitochondrial RNA reads, less than 50,000 total RNA reads were retained for further analyses. 1048 Doublets were removed using ArchR.

1050 Raw RNA counts were normalized using the NormalizeData function, scaled using the ScaleData 1051 function, and the data were visualized on a UMAP using the first 30 principal components. 1052 Dimensionality reduction for the ATAC-seq data were performed using Iterative Latent Semantic 1053 Indexing (LSI) with the addlterativeLSI function in ArchR. To impute accessibility gene scores, we 1054 used addlmputeWeights to add impute weights and plotEmbedding to visualize scores. To 1055 compare the accessibility gene scores for MYC with MYC RNA expression, getMatrixFromProject was used to extract the gene score matrix and the normalized RNA data were used.

1058 To identify variable ATAC-seq peaks on COLO320-DM and COLO320-HSR amplicons, we first calculated amplicon copy numbers based on background ATAC-seq signals as previously 1060 described, using a sliding window of five megabases moving in one-megabase increments across 1061 the reference genome ${ }^{82}$. We used the copy number $z$ scores calculated for the chr8:1240000011062129000000 interval for estimating copy numbers of MYC-bearing ecDNAs in COLO320-DM and 1063 MYC-bearing chromosomal HSRs in COLO320-HSR. We then incorporated these estimated copy 1064 numbers into the variable peak analysis as follows. COLO320-DM and COLO320-HSR cells were 1065 separately assigned into 20 bins based on their RNA expression of MYC. Next, pseudo-bulk 1066 replicates for ATAC-seq data were created using the addGroupCoverages function grouped by 1067 MYC RNA quantile bins. ATAC-seq peaks were called using addReproduciblePeakSet for each 1068 quantile bin, and peak matrices were added using addPeakMatrix. Differential peak testing was 1069 performed between the top and the bottom RNA quantile bins using getMarkerFeatures. A false 1070 discovery rate cutoff of 1e-15 was imposed. The mean copy number $z$ score for each quantile bin 1071 was then calculated and a copy number fold change between the top and bottom bin was 1072 computed. Finally, we filtered on significantly differential peaks that are located in 1073 chr8:127432631-129010071 and have fold changes above the calculated copy number fold 1074 change multiplied by 1.5 . 
Hung, Yost, Xie et al., (CHANG), p.39

1077 ChIP-seq, HiChIP and single cell multiome ATAC + gene expression data generated in this study

1078 have been deposited in GEO and are available under accession number GSE159986. GEO

1079 dataset will be made publicly available upon publication of the peer-reviewed paper.

1080

1081

\section{Code Availability}

1082 All custom code used in this work is available from the corresponding authors upon reasonable 1083 request.

1084

\section{Acknowledgements}

1086 We thank members of the Chang, Liu, Mischel, and Bafna laboratories for discussions and X. Ji, 1087 D. Wagh and J. Coller at the Stanford Functional Genomics Facility. H.Y.C. was supported by 1088 NIH R35-CA209919 and RM1-HG007735. K.L.H. was supported by the Stanford Graduate 1089 Fellowship. K.E.Y. was supported by the National Science Foundation Graduate Research 1090 Fellowship Program (NSF DGE-1656518), a Stanford Graduate Fellowship, and a $\mathrm{NCl}$ 1091 Predoctoral to Postdoctoral Fellow Transition Award (NIH F99CA253729). Cell sorting for this 1092 project was done on instruments in the Stanford Shared FACS Facility. Sequencing was 1093 performed by the Stanford Functional Genomics Facility (supported by NIH grants S10OD018220 1094 and 1S100D021763). Microscopy was performed on instruments in the UCSD Microscopy Core

1095 (supported by NINDS NS047101). Z.L. is a Janelia Group Leader and H.Y.C. and R.T. are 1096 Investigators of the Howard Hughes Medical Institute.

1098 Author Contributions

1099 K.L.H., K.E.Y., and H.Y.C. conceived the project. K.L.H., K.E.Y., L.X., S.W., J.T.L., C.V.D., K.K., 1100 J.T., and R.L. performed experiments. K.L.H., K.E.Y., L.X., S.W., J.T.L. analyzed data with input 1101 from M.R.C., J.M.G., and U.R. Q.S., J.C.R., R.T., V.B., P.S.M., Z.L., and H.Y.C. guided data 1102 analysis and provided feedback on experimental design. K.L.H., K.E.Y., and H.Y.C. wrote the 1103 manuscript with input from all authors.

\section{Competing Interests}

1106 H.Y.C. is a co-founder of Accent Therapeutics, Boundless Bio, and an advisor of 10x Genomics, 1107 Arsenal Biosciences, and Spring Discovery. P.S.M. is a co-founder of Boundless Bio, Inc. He 1108 has equity and chairs the scientific advisory board, for which he is compensated. V.B. is a co1109 founder and advisor of Boundless Bio. 
bioRxiv preprint doi: https://doi.org/10.1101/2020.11.19.390278; this version posted November 20, 2020. The copyright holder for this preprint (which was not certified by peer review) is the author/funder. All rights reserved. No reuse allowed without permission.

Hung, Yost, Xie et al., (CHANG), p.40

\section{Materials \& Correspondence}

1112 Correspondence and requests for materials should be addressed to Howard Y. Chang

1113 (howchang@stanford.edu).

1114 


\section{Supplemental Tables}

1117 Supplemental Table 1. Primer sequences. All primers used for library amplification or RT-qPCR 1118 are listed.

\begin{tabular}{|c|c|c|}
\hline primer & sequence & assay \\
\hline sgRNA_backbone_outer_fw & CAGCACAAAAGGAAACTCACCCTAACTGTAAAG & $\begin{array}{l}\text { CRISPRi_pool_I } \\
\text { ibrary_prep }\end{array}$ \\
\hline sgRNA_backbone_outer_rv & GGCCGCCTAATGGATCCTAGTACTCG & $\begin{array}{l}\text { CRISPRi_pool_I } \\
\text { ibrary_prep }\end{array}$ \\
\hline p5_mU6_0nt_stagger & $\begin{array}{l}\text { ACACTCTTTCCCTACACGACGCTCTTCCGATCTT } \\
\text { CCCTTGGAGAACCACCTTGT }\end{array}$ & $\begin{array}{l}\text { CRISPRi_pool_I } \\
\text { ibrary_prep }\end{array}$ \\
\hline p5_mU6_1nt_stagger & $\begin{array}{l}\text { ACACTCTTTCCCTACACGACGCTCTTCCGATCTC } \\
\text { TCCCTTGGAGAACCACCTTGT }\end{array}$ & $\begin{array}{l}\text { CRISPRi_pool_I } \\
\text { ibrary_prep }\end{array}$ \\
\hline p5_mU6_2nt_stagger & $\begin{array}{l}\text { ACACTCTTTCCCTACACGACGCTCTTCCGATCTG } \\
\text { CTCCCTTGGAGAACCACСTTGT }\end{array}$ & $\begin{array}{l}\text { CRISPRi_pool_I } \\
\text { ibrary_prep }\end{array}$ \\
\hline p5_mU6_3nt_stagger & $\begin{array}{l}\text { ACACTCTTTCCCTACACGACGCTCTTCCGATCTA } \\
\text { GCTCCCTTGGAGAACCACCTTGT }\end{array}$ & $\begin{array}{l}\text { CRISPRi_pool_I } \\
\text { ibrary_prep }\end{array}$ \\
\hline p7adpt_spRNAI105nt_rev & $\begin{array}{l}\text { GTGACTGGAGTTCAGACGTGTGCTCTTCCGATC } \\
\text { TGCACCGACTCGGTGCCACT }\end{array}$ & $\begin{array}{l}\text { CRISPRi_pool_I } \\
\text { ibrary_prep }\end{array}$ \\
\hline MYC_exon1_fw & AGGCTCTCCTTGCAGCTGCTTA & RT_qPCR \\
\hline MYC_exon2_rv & GCTAACGTTGAGGGGCATCGTC & RT_qPCR \\
\hline PVT1_exon1_fw & GAAAGGATGTTGGCGGTCCCTG & RT_qPCR \\
\hline total_MYC_exon2_fw & TCCACCTCCAGCTTGTACCT & RT_qPCR \\
\hline total_MYC_exon2_rv & CGTCGAGGAGAGCAGAGAAT & RT_qPCR \\
\hline GAPDH_fw & GGAGCGAGATCCCTCCAAAAT & RT_qPCR \\
\hline GAPDH_rv & GGCTGTTGTCATACTTCTCATGG & RT_qPCR \\
\hline 18S_fw & TAAAGGAATTGACGGAAGGGCA & RT_qPCR \\
\hline 18S_rv & ATCTGTCAATCCTGTCCGTGTC & RT_qPCR \\
\hline MYC_exon3_fw & GGCTCCTGGCAAAAGGTCA & RT_qPCR \\
\hline MYC_exon3_rv & CTGCGTAGTTGTGCTGATGT & RT_qPCR \\
\hline
\end{tabular}


1122 Supplemental Table 2. sgRNA sequences. All sgRNAs used in the CRISPR interference 1123 studies and ecDNA editing for TetO insertion are listed.

\begin{tabular}{|c|c|c|}
\hline gRNA_ID & sequence & info \\
\hline gRNA9 & GCCTCCGGGCAGAGCGCGTG & PVT1_TSS \\
\hline gRNA10 & GGAATAGGGGGCTTCGCCTC & MYC_TSS \\
\hline gRNA11 & GATGGATGGACCACAACAGGG & COLO320DM_MYC_enhancer \\
\hline gRNA12 & GGTTTCCTTATCTATGAACCG & COLO320DM_MYC_enhancer \\
\hline gRNA13 & GAGTGCATTAGAGGTACACAG & COLO320DM_MYC_enhancer \\
\hline gRNA14 & GATAAAAAGTGCCCGACAATG & COLO320DM_MYC_enhancer \\
\hline gRNA15 & GGGTTGGACTGATGACCTCAG & COLO320DM_MYC_enhancer \\
\hline gRNA16 & GAGGTTTGGAGACATACACAT & COLO320DM_MYC_enhancer \\
\hline gRNA22 & GTCTTAACATAGGTGCTACCA & MYC_ecDNA_element \\
\hline gRNA23 & GAAAAAGTAAGAAAAATGCCG & MYC_ecDNA_element \\
\hline gRNA24 & GCTCCACAGAACCCAGGACAA & MYC_ecDNA_element \\
\hline gRNA25 & GATGACCCCTGACCTAGAGT & MYC_ecDNA_element \\
\hline gRNA26 & GTGTCTACTCTACCACAACA & MYC_ecDNA_element \\
\hline gRNA27 & GCCCACAAGTTATCTACCGGG & MYC_ecDNA_element \\
\hline gRNA28 & GATGCCAATGCTTGCTCAAGG & MYC_ecDNA_element \\
\hline gRNA29 & GAATGTAAACCTTAACAACCC & MYC_ecDNA_element \\
\hline gRNA30 & GAATAGAGTGGCCTTGAACG & MYC_ecDNA_element \\
\hline gRNA31 & GATGACGGTTATAGAGCCCTA & MYC_ecDNA_element \\
\hline gRNA32 & GCTACATAATTCGGTTCAGCT & MYC_ecDNA_element \\
\hline gRNA33 & GCGGCCAGGAGTGTCTATCAG & MYC_ecDNA_element \\
\hline gRNA34 & GCAAGGCAGTTAACCAAACCC & MYC_ecDNA_element \\
\hline gRNA35 & GTAAGTACCCTACTCTCTACG & MYC_ecDNA_element \\
\hline gRNA36 & GCATTTGAAAGCATTAACCCA & MYC_ecDNA_element \\
\hline gRNA37 & GTCGCGCCTGGATGTCAACGA & MYC_ecDNA_element \\
\hline gRNA38 & GCGCGCGTAGTTAATTCATG & MYC_ecDNA_element \\
\hline gRNA39 & GAGAGCGGCTAGGGCGCGAGT & MYC_ecDNA_element \\
\hline gRNA40 & GTAGTCTGAATCACTAAGTCC & MYC_ecDNA_element \\
\hline gRNA41 & GACCATGTGCTCATCCGTCAT & MYC_ecDNA_element \\
\hline gRNA42 & GGTTGCCCGTGACGTCACGG & MYC_ecDNA_element \\
\hline gRNA43 & GACAGCTGGGTTAGACAGATA & MYC_ecDNA_element \\
\hline gRNA44 & GAGTGGACATAGGACTAGACA & MYC_ecDNA_element \\
\hline gRNA45 & GTGAGTGATGACCCTGAAGTG & MYC_ecDNA_element \\
\hline gRNA54 & GGCTCGCGGTAGGGACACGT & FGFR2_ecDNA_element \\
\hline
\end{tabular}




\begin{tabular}{|l|l|l|}
\hline gRNA55 & GTGATAGATTATTCCCACACA & FGFR2_ecDNA_element \\
\hline gRNA56 & GTCTGGAATGATGAAGACCCA & FGFR2_ecDNA_element \\
\hline gRNA57 & GAGGCCTCCAGCAGACTAGCA & FGFR2_ecDNA_element \\
\hline gRNA58 & GACTGTGTAGTTACAAGCTGT & FGFR2_ecDNA_element \\
\hline gRNA59 & GTTTATTGGGTGTTAACCGT & FGFR2_ecDNA_element \\
\hline gRNA60 & GAATGAGCTGCAGAATAAGAG & FGFR2_ecDNA_element \\
\hline gRNA61 & GGGTAGCAGCAAATAAATGA & FGFR2_ecDNA_element \\
\hline gRNA62 & GCACACGCAACAGCTACAAAG & FGFR2_ecDNA_element \\
\hline gRNA63 & GACACCAACTGCCAACCACAT & FGFR2_ecDNA_element \\
\hline gRNA64 & GAGTCTCATAAATCAAGACAG & FGFR2_ecDNA_element \\
\hline gRNA65 & GTAAGTCATATGATATAGACT & FGFR2_ecDNA_element \\
\hline gRNA66 & GAGGAGCACAGTATGTAAGCA & FGFR2_ecDNA_element \\
\hline gRNA67 & GTTTGACTCTCAGGAGTCCTG & FGFR2_ecDNA_element \\
\hline gRNA68 & GAATTGTTGTTGGGTACCAA & FGFR2_ecDNA_element \\
\hline gRNA69 & GCTGGGATTAAATCAGACCT & FGFR2_ecDNA_element \\
\hline gRNA70 & GTAGTCCTTAAATCACTCCCG & FGFR2_ecDNA_element \\
\hline gRNA71 & GTGGAGCGTCCATTATGGTG & FGFR2_ecDNA_element \\
\hline gRNA72 & GCTAGTTCCAAGGATGCGGTG & FGFR2_ecDNA_element \\
\hline gRNA73 & GGCATCCTTGTTAGCGACCA & FGFR2_ecDNA_element \\
\hline gRNA74 & GCCCCAACTCGCAGTAACGC & FGFR2_ecDNA_element \\
\hline gRNA75 & GAGAAGAGCCATCCATCAGA & FGFR2_ecDNA_element \\
\hline gRNA76 & GCGCGGAAGGACTAGCATTG & FGFR2_ecDNA_element \\
\hline gRNA77 & GTGCAGATGATGAGCGACCC & FGFR2_ecDNA_element \\
\hline MYC_sgRNA & GGAGAGCTTGTGGACCGAGC & MYC_ecDNA_TetO_insertion \\
\hline
\end{tabular}


Hung, Yost, Xie et al., (CHANG), p.44

1126

1127

1128

1129

1130

1131

1132

1133

1134

1135

1136

1137

1138

1139

1140

1141

1142

1143

1144

1145

1146

1147

1148

1149

1150

1151

1152

1153

1154

1155

1156

1157

1158

1159

1160

1161

1162

1163

1164

1165

1166

1167

1168

1169

1170

1171

1172

1173

1174

1175

1176

\section{REFERENCES}

1. Wu, S. et al. Circular ecDNA promotes accessible chromatin and high oncogene expression. Nature 1-5 (2019) doi:10.1038/s41586-019-1763-5.

2. Gorkin, D. U., Leung, D. \& Ren, B. The 3D Genome in Transcriptional Regulation and Pluripotency. Cell Stem Cell 14, 762-775 (2014).

3. Zheng, H. \& Xie, W. The role of 3D genome organization in development and cell differentiation. Nature Reviews Molecular Cell Biology 20, 535-550 (2019).

4. Bailey, C., Shoura, M. J., Mischel, P. S. \& Swanton, C. Extrachromosomal DNA - relieving heredity constraints, accelerating tumour evolution. Annals of Oncology (2020) doi:10.1016/j.annonc.2020.03.303.

5. Kim, H. et al. Extrachromosomal DNA is associated with oncogene amplification and poor outcome across multiple cancers. Nature Genetics 52, 891-897 (2020).

6. Turner, K. M. et al. Extrachromosomal oncogene amplification drives tumour evolution and genetic heterogeneity. Nature 543, 122-125 (2017).

7. Verhaak, R. G. W., Bafna, V. \& Mischel, P. S. Extrachromosomal oncogene amplification in tumour pathogenesis and evolution. Nature Reviews Cancer 19, 283 (2019).

8. van der Bliek, A. M., Lincke, C. R. \& Borst, P. Circular DNA of 3T6R50 double minute chromosomes. Nucleic Acids Research 16, 4841-4851 (1988).

9. Hamkalo, B. A., Farnham, P. J., Johnston, R. \& Schimke, R. T. Ultrastructural features of minute chromosomes in a methotrexate-resistant mouse 3T3 cell line. Proceedings of the National Academy of Sciences 82, 1126-1130 (1985).

10. Maurer, B. J., Lai, E., Hamkalo, B. A., Hood, L. \& Attardi, G. Novel submicroscopic extrachromosomal elements containing amplified genes in human cells. Nature 327, 434437 (1987).

11. VanDevanter, D. R., Piaskowski, V. D., Casper, J. T., Douglass, E. C. \& Von Hoff, D. D. Ability of Circular Extrachromosomal DNA Molecules to Carry Amplified MYCN Protooncogenes in Human Neuroblastomas In Vivo. J Natl Cancer Inst 82, 1815-1821 (1990).

12. Nathanson, D. A. et al. Targeted Therapy Resistance Mediated by Dynamic Regulation of Extrachromosomal Mutant EGFR DNA. Science 343, 72-76 (2014).

13. Ståhl, F., Wettergren, Y. \& Levan, G. Amplicon structure in multidrug-resistant murine cells: a nonrearranged region of genomic DNA corresponding to large circular DNA. Molecular and Cellular Biology 12, 1179-1187 (1992).

14. Vicario, R. et al. Patterns of HER2 Gene Amplification and Response to Anti-HER2 Therapies. PLOS ONE 10, e0129876 (2015).

15. Carroll, S. M. et al. Double minute chromosomes can be produced from precursors derived from a chromosomal deletion. Molecular and Cellular Biology 8, 1525-1533 (1988).

16. Kitajima, K., Haque, M., Nakamura, H., Hirano, T. \& Utiyama, H. Loss of Irreversibility of Granulocytic Differentiation Induced by Dimethyl Sulfoxide in HL-60 Sublines with a Homogeneously Staining Region. Biochemical and Biophysical Research Communications 288, 1182-1187 (2001).

17. Quinn, L. A., Moore, G. E., Morgan, R. T. \& Woods, L. K. Cell Lines from Human Colon Carcinoma with Unusual Cell Products, Double Minutes, and Homogeneously Staining Regions. Cancer Research 39, 4914-4924 (1979).

18. Storlazzi, C. T. et al. Gene amplification as double minutes or homogeneously staining regions in solid tumors: Origin and structure. Genome Res. 20, 1198-1206 (2010).

19. Wahl, G. M. The Importance of Circular DNA in Mammalian Gene Amplification. Cancer Res 49, 1333-1340 (1989).

20. Kumar, P. et al. ATAC-seq identifies thousands of extrachromosomal circular DNA in cancer and cell lines. Science Advances 6, eaba2489 (2020). 
Hung, Yost, Xie et al., (CHANG), p.45

1177

1178

1179

1180

1181

1182

1183

1184

1185

1186

1187

1188

1189

1190

1191

1192

1193

1194

1195

1196

1197

1198

1199

1200

1201

1202

1203

1204

1205

1206

1207

1208

1209

1210

1211

1212

1213

1214

1215

1216

1217

1218

1219

1220

1221

1222

1223

1224

1225

1226

21. Morton, A. R. et al. Functional Enhancers Shape Extrachromosomal Oncogene Amplifications. Cell 0, (2019).

22. Itoh, N. \& Shimizu, N. DNA replication-dependent intranuclear relocation of double minute chromatin. Journal of Cell Science 111 ( Pt 22), 3275-3285 (1998).

23. Kanda, T., Sullivan, K. F. \& Wahl, G. M. Histone-GFP fusion protein enables sensitive analysis of chromosome dynamics in living mammalian cells. Current Biology 8, 377-385 (1998).

24. Oobatake, Y. \& Shimizu, N. Double-strand breakage in the extrachromosomal double minutes triggers their aggregation in the nucleus, micronucleation, and morphological transformation. Genes, Chromosomes and Cancer 59, 133-143 (2020).

25. Beroukhim, R. et al. The landscape of somatic copy-number alteration across human cancers. Nature 463, 899-905 (2010).

26. Cho, S. W. et al. Promoter of IncRNA Gene PVT1 Is a Tumor-Suppressor DNA Boundary Element. Cell 173, 1398-1412.e22 (2018).

27. Graham, M. \& Adams, J. m. Chromosome 8 breakpoint far 3' of the c-myc oncogene in a Burkitt's lymphoma 2;8 variant translocation is equivalent to the murine pvt-1 locus. The EMBO Journal 5, 2845-2851 (1986).

28. Graham, M., Adams, J. M. \& Cory, S. Murine T lymphomas with retroviral inserts in the chromosomal 15 locus for plasmacytoma variant translocations. Nature $314,740-743$ (1985).

29. Beliveau, B. J. et al. Versatile design and synthesis platform for visualizing genomes with Oligopaint FISH probes. Proceedings of the National Academy of Sciences 109, 2130121306 (2012).

30. McInnes, L., Healy, J. \& Melville, J. UMAP: Uniform Manifold Approximation and Projection for Dimension Reduction. arXiv:1802.03426 [cs, stat] (2020).

31. Granja, J. M. et al. ArchR: An integrative and scalable software package for single-cell chromatin accessibility analysis. bioRxiv 2020.04.28.066498 (2020) doi:10.1101/2020.04.28.066498.

32. Corces, M. R. et al. The chromatin accessibility landscape of primary human cancers. Science 362, (2018).

33. Deshpande, V. et al. Exploring the landscape of focal amplifications in cancer using AmpliconArchitect. Nat Commun 10, 1-14 (2019).

34. Hann, S. R., King, M. W., Bentley, D. L., Anderson, C. W. \& Eisenman, R. N. A non-AUG translational initiation in c-myc exon 1 generates an $\mathrm{N}$-terminally distinct protein whose synthesis is disrupted in Burkitt's lymphomas. Cell 52, 185-195 (1988).

35. Hann, S. R., Dixit, M., Sears, R. C. \& Sealy, L. The alternatively initiated c-Myc proteins differentially regulate transcription through a noncanonical DNA-binding site. Genes Dev. 8 , 2441-2452 (1994).

36. Alitalo, K., Schwab, M., Lin, C. C., Varmus, H. E. \& Bishop, J. M. Homogeneously staining chromosomal regions contain amplified copies of an abundantly expressed cellular oncogene (c-myc) in malignant neuroendocrine cells from a human colon carcinoma. Proc Natl Acad Sci U S A 80, 1707-1711 (1983).

37. Schwab, M., Klempnauer, K. H., Alitalo, K., Varmus, H. \& Bishop, M. Rearrangement at the 5 ' end of amplified c-myc in human COLO 320 cells is associated with abnormal transcription. Mol Cell Biol 6, 2752-2755 (1986).

38. Shtivelman, E. \& Bishop, J. M. The PVT gene frequently amplifies with MYC in tumor cells. Mol Cell Biol 9, 1148-1154 (1989).

39. L'Abbate, A. et al. Genomic organization and evolution of double minutes/homogeneously staining regions with MYC amplification in human cancer. Nucleic Acids Research 42, 9131-9145 (2014). 
40. Mumbach, M. R. et al. HiChIP: efficient and sensitive analysis of protein-directed genome architecture. Nature Methods 13, 919 (2016).

41. Mumbach, M. R. et al. Enhancer connectome in primary human cells identifies target genes of disease-associated DNA elements. Nat. Genet. 49, 1602-1612 (2017).

42. Parker, S. C. J. et al. Chromatin stretch enhancer states drive cell-specific gene regulation and harbor human disease risk variants. PNAS 110, 17921-17926 (2013).

43. Whyte, W. A. et al. Master Transcription Factors and Mediator Establish Super-Enhancers at Key Cell Identity Genes. Cell 153, 307-319 (2013).

44. Lovén, J. et al. Selective Inhibition of Tumor Oncogenes by Disruption of Super-Enhancers. Cell 153, 320-334 (2013).

45. Filippakopoulos, P. et al. Selective inhibition of BET bromodomains. Nature 468, 1067-1073 (2010).

46. Sabari, B. R. et al. Coactivator condensation at super-enhancers links phase separation and gene control. Science 361, eaar3958 (2018).

47. Fulco, C. P. et al. Systematic mapping of functional enhancer-promoter connections with CRISPR interference. Science 354, 769-773 (2016).

48. Rajkumar, U. et al. EcSeg: Semantic Segmentation of Metaphase Images Containing Extrachromosomal DNA. iScience 21, 428-435 (2019).

49. Xue, K. S., Hooper, K. A., Ollodart, A. R., Dingens, A. S. \& Bloom, J. D. Cooperation between distinct viral variants promotes growth of H3N2 influenza in cell culture. eLife $\mathbf{5}$, e13974 (2016).

50. Vignuzzi, M., Stone, J. K., Arnold, J. J., Cameron, C. E. \& Andino, R. Quasispecies diversity determines pathogenesis through cooperative interactions in a viral population. Nature 439, 344-348 (2006).

51. Nikolaev, S. et al. Extrachromosomal driver mutations in glioblastoma and low-grade glioma. Nature Communications 5, 5690 (2014).

52. Cisse, I. I. et al. Real-Time Dynamics of RNA Polymerase II Clustering in Live Human Cells. Science 341, 664-667 (2013).

53. Furlong, E. E. M. \& Levine, M. Developmental enhancers and chromosome topology. Science 361, 1341-1345 (2018).

54. Hnisz, D., Shrinivas, K., Young, R. A., Chakraborty, A. K. \& Sharp, P. A. A Phase Separation Model for Transcriptional Control. Cell 169, 13-23 (2017).

55. McSwiggen, D. T., Mir, M., Darzacq, X. \& Tjian, R. Evaluating phase separation in live cells: diagnosis, caveats, and functional consequences. Genes Dev. 33, 1619-1634 (2019).

56. Chong, S. et al. Imaging dynamic and selective low-complexity domain interactions that control gene transcription. Science 361, (2018).

57. Li, J. et al. Single-gene imaging links genome topology, promoter-enhancer communication and transcription control. Nature Structural \& Molecular Biology 1-9 (2020) doi:10.1038/s41594-020-0493-6.

58. Gibson, B. A. et al. Organization of Chromatin by Intrinsic and Regulated Phase Separation. Cell 179, 470-484.e21 (2019).

59. Rosencrance, C. D. et al. Chromatin Hyperacetylation Impacts Chromosome Folding by Forming a Nuclear Subcompartment. Mol Cell 78, 112-126.e12 (2020).

60. McSwiggen, D. T. et al. Evidence for DNA-mediated nuclear compartmentalization distinct from phase separation. eLife 8, e47098 (2019).

61. Shin, Y. et al. Liquid Nuclear Condensates Mechanically Sense and Restructure the Genome. Cell 175, 1481-1491.e13 (2018).

62. Risca, V. I., Denny, S. K., Straight, A. F. \& Greenleaf, W. J. Variable chromatin structure revealed by in situ spatially correlated DNA cleavage mapping. Nature 541, 237-241 (2017). 
63. You, J., Croyle, J. L., Nishimura, A., Ozato, K. \& Howley, P. M. Interaction of the Bovine Papillomavirus E2 Protein with Brd4 Tethers the Viral DNA to Host Mitotic Chromosomes. Cell 117, 349-360 (2004).

64. Cortez, J. T. et al. CRISPR screen in regulatory T cells reveals modulators of Foxp3. Nature 582, 416-420 (2020).

65. Li, W. et al. MAGeCK enables robust identification of essential genes from genome-scale CRISPR/Cas9 knockout screens. Genome Biology 15, 554 (2014).

66. Xie, L. et al. 3D ATAC-PALM: super-resolution imaging of the accessible genome. Nature Methods 1-7 (2020) doi:10.1038/s41592-020-0775-2.

67. Normanno, D. et al. Probing the target search of DNA-binding proteins in mammalian cells using TetR as model searcher. Nature Communications 6, 7357 (2015).

68. Veatch, S. L. et al. Correlation Functions Quantify Super-Resolution Images and Estimate Apparent Clustering Due to Over-Counting. PLOS ONE 7, e31457 (2012).

69. Ghandi, M. et al. Next-generation characterization of the Cancer Cell Line Encyclopedia. Nature 569, 503-508 (2019).

70. Mumbach, M. R. et al. HiChIRP reveals RNA-associated chromosome conformation. Nature Methods 16, 489-492 (2019).

71. Sedlazeck, F. J. et al. Accurate detection of complex structural variations using singlemolecule sequencing. Nature Methods 15, 461-468 (2018).

72. Haas, B. J. et al. Accuracy assessment of fusion transcript detection via read-mapping and de novo fusion transcript assembly-based methods. Genome Biology 20, 213 (2019).

73. Hahne, F. \& Ivanek, R. Visualizing Genomic Data Using Gviz and Bioconductor. in Statistical Genomics: Methods and Protocols (eds. Mathé, E. \& Davis, S.) 335-351 (Springer, 2016). doi:10.1007/978-1-4939-3578-9_16.

74. Langmead, B. \& Salzberg, S. L. Fast gapped-read alignment with Bowtie 2. Nature Methods 9, 357-359 (2012).

75. Bolger, A. M., Lohse, M. \& Usadel, B. Trimmomatic: a flexible trimmer for Illumina sequence data. Bioinformatics 30, 2114 (2014).

76. Zhang, Y. et al. Model-based analysis of ChIP-Seq (MACS). Genome Biol. 9, R137 (2008).

77. Ramírez, F. et al. deepTools2: a next generation web server for deep-sequencing data analysis. Nucleic Acids Res 44, W160-W165 (2016).

78. Servant, N. et al. HiC-Pro: an optimized and flexible pipeline for $\mathrm{Hi}-\mathrm{C}$ data processing. Genome Biol. 16, 259 (2015).

79. Bhattacharyya, S., Chandra, V., Vijayanand, P. \& Ay, F. Identification of significant chromatin contacts from HiChIP data by FitHiChIP. Nature Communications 10, 4221 (2019).

80. Rao, S. S. P. et al. A 3D Map of the Human Genome at Kilobase Resolution Reveals Principles of Chromatin Looping. Cell 159, 1665-1680 (2014).

81. Butler, A., Hoffman, P., Smibert, P., Papalexi, E. \& Satija, R. Integrating single-cell transcriptomic data across different conditions, technologies, and species. Nature Biotechnology 36, 411-420 (2018).

82. Satpathy, A. T. et al. Massively parallel single-cell chromatin landscapes of human immune cell development and intratumoral T cell exhaustion. Nat Biotechnol 37, 925-936 (2019). 